Revista de Matemática: Teoría y Aplicaciones 2001 8(2): 101-130

CIMPA - UCR - CCSS ISSN: 1409-2433

\title{
TEORÍA DE NUDOS GEOMÉTRICOS E ISOTOPÍA POLIGONAL
}

\author{
Jorge Alberto Calvo Soto*
}

Recibido: 25 Enero 2000

\begin{abstract}
Resumen
El espacio de los polígonos de $n$ lados, inmersos en el espacio euclídeo de tres dimensiones, consiste de una variedad suave en la cual los puntos corresponden a nudos lineales a trozos o "geométricos", mientras que los arcos corresponden a isotopías que preservan la estructura geométrica de esos nudos. Se describe la topología de estos espacios para los casos $n=6$ y $n=7$. En ambos casos, cada espacio consta de cinco componentes, aunque contiene sólo tres (cuando $n=6$ ) o cuatro (cuando $n=7$ ) tipos topológicos de nudos. Por lo tanto la "equivalencia geométrica de nudos" es estrictamente más fuerte que la equivalencia topológica. Este hecho se demuestra con el nudo trébol hexagonal y el nudo doble heptagonal, los cuales, a diferencia de sus contrapartes topológicas, no son reversibles. Se discutirán también las extensiones de estos resultados a $\operatorname{los} \operatorname{casos} n \geq 8$.
\end{abstract}

Palabras clave: nudos poligonales, polígonos espaciales, espacios de nudos, invariantes de nudos.

\begin{abstract}
The space of $n$-sided polygons embedded in euclidean three-space consists of a smooth manifold in which points correspond to piecewise linear or "geometric" knots, while paths correspond to isotopies which preserve the geometric structure of these knots. The topology of these spaces for the case $n=6$ and $n=7$ is described. In both of these cases, each knot space consists of five components, but contains only three (when $n=6$ ) or four (when $n=7$ ) topological knot types. Therefore "geometric knot equivalence" is strictly stronger than topological equivalence. This point is demonstrated by the hexagonal trefoils and heptagonal figure-eight knots, which, unlike their topological counterparts, are not reversible. Extending these results to the cases $n \geq 8$ will also be discussed.
\end{abstract}

Keywords: polygonal knots, space polygons, knot spaces, knot invariants.

AMS Subject Classification: 57M25

*Department of Mathematics, North Dakota State University, Fargo, ND 58105 U.S.A.

email: jorge_calvo@ndsu.nodak.edu 


\section{Introducción}

La historia del nudo es talvez tan antigua como la del hombre. Los peruanos precolombinos, por ejemplo, utilizaban el "quipo," una cuerdita con varios nudos, como especie de ábaco [3]. Además, tenemos al historiador latino Quinto Curcio Rufo, quien nos cuenta cómo Alejandro Magno, empeñado en que se cumpliera el oráculo que le prometía el dominio del Asia, cortó el nudo gordiano al no poder desatarlo [7]. Sin embargo, el estudio sistemático de los nudos no comenzó hasta finales del siglo XIX, empujado en parte por la teoría de Lord Kelvin en la cual los átomos eran vórtices anudados en el éter. Aún así, por casi cien años la teoría matemática de nudos se consideró poco más que una colección de ejemplos topológicos y algebraicos con variadas y extrañas propiedades. No fue hasta las décadas de los sesentas y setentas que varios resultados importantes pusieron a la teoría de nudos al centro de la topología tridimensional moderna. Entre estos se merece mencionar:

- el teorema de Lickorish y Wallace, según el cual toda variedad topológica tridimensional se obtiene como el resultado de alguna cirugía en una colección de nudos en $\mathbb{S}^{3}[16,32]$

- el teorema de Gordon y Luecke, que asegura que dos nudos en $\mathbb{S}^{3}$ son equivalentes si y sólo si sus complementos son homeomórficos [9];

- la conjetura de geometrización de Thurston y su programa para completar de una forma definitiva una enumeración de las variedades topológicas de tres dimensiones $[28,29,30]$;

- el descubrimiento de los polinomios invariantes de Jones, HOMFLY y sus sucesores, los cuales facilitan el reconocimiento de ciertos tipos de nudos, a la vez que abren varias conecciones con la física y la mecánica estadística [11, 12].

El objetivo principal de este artículo es dar una reseña de la teoría de nudos, específicamente cuando se refiere a aquellos nudos "geométricos" que se construyen a base de trozos lineales. La próxima sección presenta un vocabulario mínimo de la teoría clásica de nudos topológicos, mientras que la Sección 3 dirige nuestra atención hacia los nudos geométricos. Esta culmina con el Teorema 1, el cual delinea el estado presente de la clasificación de los polígonos de bajo número de lados inmersos en $\mathbb{R}^{3}$. Las siguientes dos secciones exponen dos de los ingredientes claves que facilitan la prueba de este teorema. La Sección 4 trata de un método para descomponer el espacio de $n$-gonos (al cual llamaremos

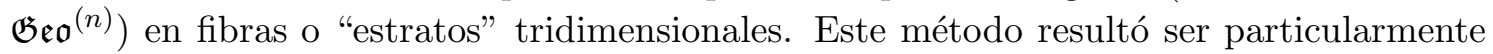
útil en el análisis de los espacios de hexágonos y heptágonos $\left({\mathfrak{G} e \mathfrak{o}^{(6)}}_{\text {y }} \mathfrak{G e o}^{(7)}\right)$. Luego, la Sección 5 describe una cota superior para el número mínimo de cruces de un nudo poligonal con $n$ segmentos. Esta cota, la cual se obtiene a través de una proyección especial del polígono a una esfera, mejora por un término lineal aquélla previamente conocida, y nos da suficiente control para clasificar los tipos topológicos de nudos presentes en el espacio de nudos octogonales $\left(\mathfrak{G} \mathfrak{e o}^{(8)}\right)$.

\section{Nudos topológicos}

Se le llama nudo a la imagen de una inmersión del círculo $\mathbb{S}^{1}$ en una variedad topológica tridimensional, tradicionalmente $\mathbb{R}^{3}$ o bien su compactificación con un punto, $\mathbb{S}^{3}$. O sea que, cuando le amarramos el cordón a un zapato, no obtenemos un "nudo" - pues todavía 
se puede desamarrar - a menos de que unamos ambos extremos del cordón y formemos un círculo anudado. En este caso el "nudo" consiste en una curva infinitésimamente delgada ubicada justamente en el centro del cordón.

Se dice que dos inmersiones $K, K^{\prime}$ son equivalentes si existe una isotopía ambiental de $\mathbb{R}^{3}$ $\left(\right.$ o $\left.\mathbb{S}^{3}\right)$ transladando una inmersión a la otra. Podemos visualizar una isotopía de este tipo como una deformación que nos lleva desde $K$ hasta $K^{\prime}$, la cual no permite que ninguna sección del nudo atraviese ninguna otra, ni que se contraiga ninguna parte esencial del nudo a un solo punto. O sea, esta deformación debe no sólo preservar la continuidad del cordón, sino también prevenir que el nudo se soque demasiado. Informalmente, decimos que dos inmersiones equivalentes representan al "mismo nudo."

La forma más común de expresar la manera en que un nudo se ubica en $\mathbb{R}^{3}$ o $\mathbb{S}^{3}$ es a través de una proyección ortogonal del nudo a un plano o esfera. Al escoger una inmersión genérica, podemos garantizar que la imagen de ésta se intersecta a sí misma transversalmente, y solamente en un número finito de puntos dobles, a los cuales se les llama cruces. Uno de los arcos incidentes a una cruz se dibuja entero mientras que al otro se le borra un poco antes y después del punto doble, para indicar que el primero pasa encima del segundo. Así, el diagrama en la Figura 1 describe un objeto realmente tridimensional.

En 1926, Kurt Reidemeister probó que toda isotopía ambiental se puede llevar a cabo como una secuencia de movidas de tres tipos [25]. Ver la Figura 2. Cada una de estas movidas hace un cambio local en la proyección del nudo, y se supone que no afectan a la proyección lejos del área indicada.

A estas tres movidas se les puede agregar una deformación plana que simplemente le cambia el aspecto a la proyección, pero no su esencia. Por ejemplo, regresemos al nudo de la Figura 1: Al utilizar una movida de tipo II, eliminamos las dos cruces a la derecha extrema. Una segunda movida II aniquila las dos cruces superiores. Luego, una movida III saca una de las cruces interiores para formar un "lazo nugatorio," el cual eliminamos con una movida de tipo I. Finalmente, una movida de tipo II, seguida por una de tipo I, nos lleva a una proyección sin cruces. Ver la Figura 3. Ahora una deformación plana convierte esta curva en un círculo estándar. Todo esto nos viene a decir que el nudo en la Figura 1 no estaba realmente anudado. Por lo tanto, se le llama a éste nudo trivial. ${ }^{1}$

\footnotetext{
1 En Inglés, también se usa la palabra "unknot," que se traduce, más o menos, a "no nudo."
}

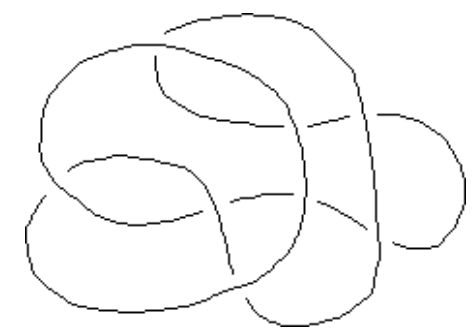

Figura 1. La proyección de un nudo con 8 cruces. 
Tipo I:

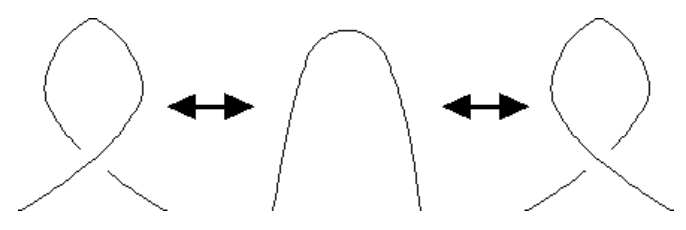

Tipo II:

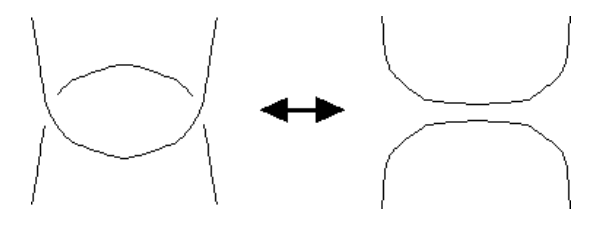

Tipo III:

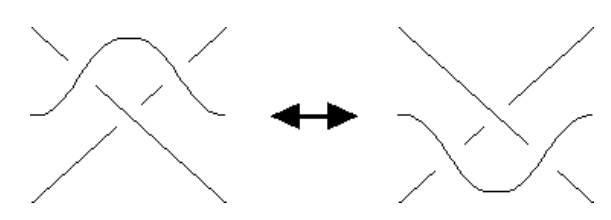

Figura 2. Las movidas de Reidemeister.

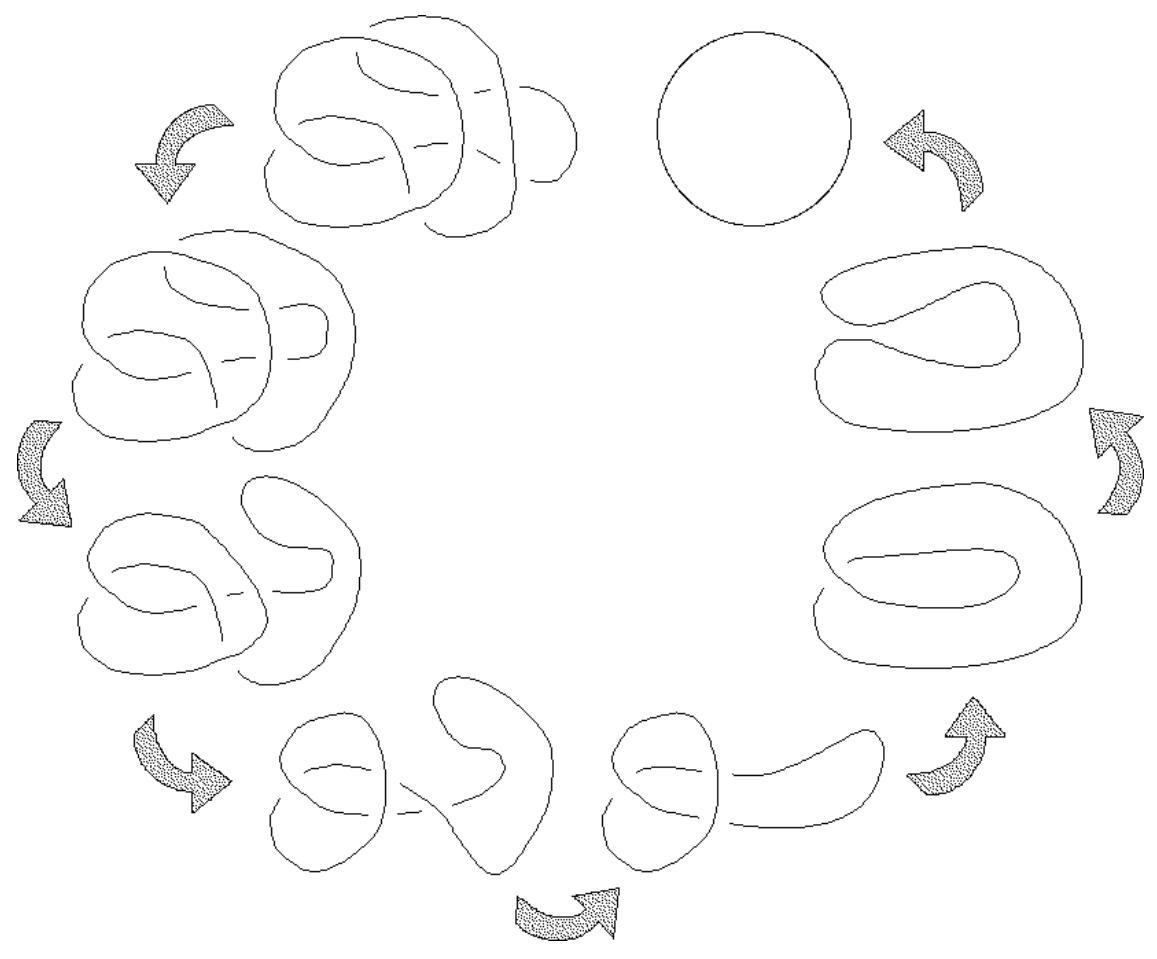

Figura 3. Isotopía de un nudo trivial. 
La Figura 4 indica dos de los nudos más simples que están realmente anudados: el nudo trébol y el nudo doble. ${ }^{2}$ Unos de los primeros teoremas en la teoría de nudos consisten en probar que estos dos nudos no son equivalentes ni al trivial, ni uno al otro.

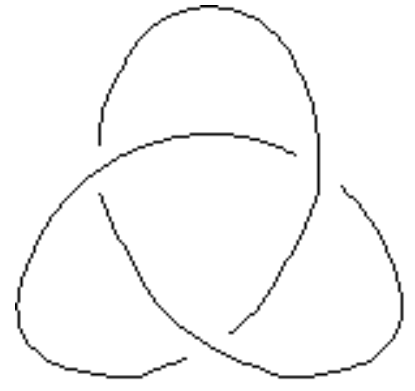

(a) nudo trébol

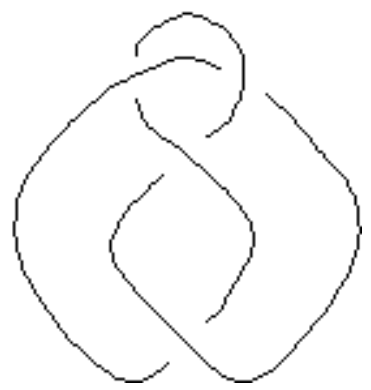

(b) nudo doble

Figura 4. Proyecciones con mínimo número de cruces de los nudos trébol y doble.

Es importante notar que las proyecciones dadas en la Figura 4 utilizan el número mínimo de cruces posibles: tres en el caso del trébol y cuatro en el caso del doble. A finales del siglo XIX, Thomas Kirkman, C. N. Little y Peter Tait comenzaron la difícil tarea de enumerar los nudos "primos" mas simples $[13,15,18,27] .{ }^{3}$ El orden en que aparecen los nudos en esta enumeración es poco más que arbitrario ya que, para cada número de cruces, sólo el primer par de nudos sigue un patrón bien definido. ${ }^{4}$ No obstante, tomamos ésta como la "lista canónica" de nudos primos, y la utilizamos para darle nombre a todo nudo que forma parte de ella, de acuerdo con la notación Alexader-Briggs [2]. Según ésta, ya que el trébol y el doble son los primeros (en realidad los únicos) nudos de tres y cuatro cruces, respectivamente, se les denota como los nudos $3_{1}$ y $4_{1}$; de la misma manera, el nudo $8_{10}$ consiste en el décimo nudo con sólo ocho cruces en la lista canónica de Tait, Kirkman y Little.

El trébol que aparece en la Figura 4(a) es "derecho," o sea que todas sus cruces siguen la "regla de la mano derecha." Para ésto, supongamos que le damos al nudo una orientación, como la dirección de la trayectoria de una hormiga que viaja continuamente alrededor del nudo. Decimos que una cruz sigue la regla de la mano derecha si, al apuntar el dedo pulgar de la mano derecha en dirección del arco superior de la cruz, los otros dedos se enroscan en dirección del arco inferior. Ver la Figura 5.

Si tomamos un nudo y lo reflejamos a través de un plano, obtendremos su anverso. En el caso del trébol derecho de la Figura 4(a), éste será un trébol izquierdo, como el que aparece en la Figura 6(b). Métodos avanzados, como el uso de polinomios invariantes, nos permiten probar que éstos dos tréboles no son equivalentes. Por esta razón decimos que

\footnotetext{
2 En Inglés, al trébol se le llama "trefoil knot" o "overhand knot," mientras que al doble se le llama "figure-eight knot."

3 Se le llama a un nudo primo si toda esfera en $\mathbb{S}^{3}$ que intersecte el nudo transversalmente en dos puntos separa a $\mathbb{S}^{3}$ en dos bolas, una de las cuales intersecta el nudo en un arco trivial homeomórfico al eje polar. La idea aquí es que un nudo primo no se puede "factorizar" en dos nudos más simples, a menos de que uno de ellos sea el trivial.

${ }^{4}$ Ver, por ejemplo, los apéndices de los textos clásicos de la teoría de nudos $[1,14,17,26]$.
} 

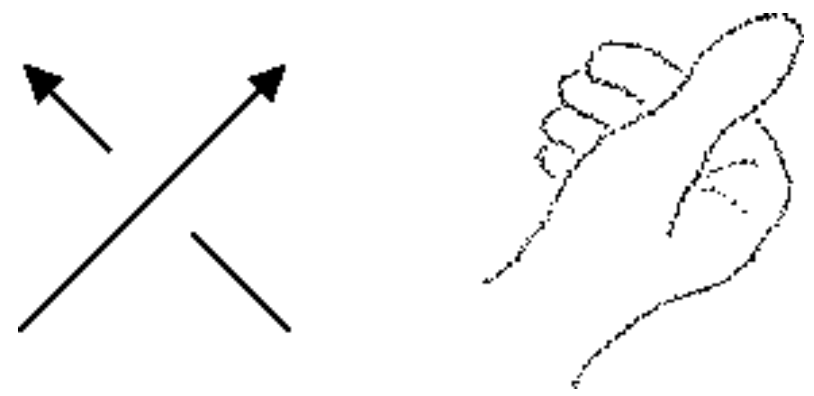

FigurA 5. La regla de la mano derecha.

el trébol es un nudo quiral, de la palabra griega que significa "mano." Por convención, usaremos la nomenclatura de " $3_{1}$ " para referirnos al trébol derecho, y " $-3_{1}$ " para referirnos al izquierdo.

Como contraste tomemos el anverso del nudo doble que aparece en la Figura 4(b). Este es equivalente al original, como resulta fácil de comprobar ya sea usando una cuerda o una secuencia de movidas de Reidemeister; pero más fácil aún es notar que las dos proyecciones serían exactamente la misma si proyectáramos no en un plano sino en una esfera grande. Esto es equivalente a identificar el plano de proyección con el plano complejo $\mathbb{C}$ y aplicar una transformación Möbius que cambie la ubicación del punto al infinito, colocándolo en una de las regiones triangulares en el complemento de la proyección. Por lo tanto, decimos que el nudo doble es aquiral. ${ }^{5}$

Si tomamos los nudos de la Figura 4 y los rotamos por un ángulo de $\pi$ alrededor de un eje vertical, obtendremos un par de proyecciones equivalentes a las originales. Por ejemplo, si observamos el comportamiento de la cruz inferior en cada diagrama durante esta rotación, notaremos que el arco que originalmente pasa por encima de la cruz pasará por debajo de la cruz después de ser rotado. Sin embargo, este arco que antes cruzaba de noroeste a sureste, ahora cruzará de suroeste a noreste. Por lo tanto, aún después de la rotación, esta cruz será derecha! No obstante, si le damos una orientación en particular a

\footnotetext{
5 Ciertos autores también usan la palabra "anfiquiral," pero por consideración a las aplicaciones a la química se hace de éste un término arcaico.
}

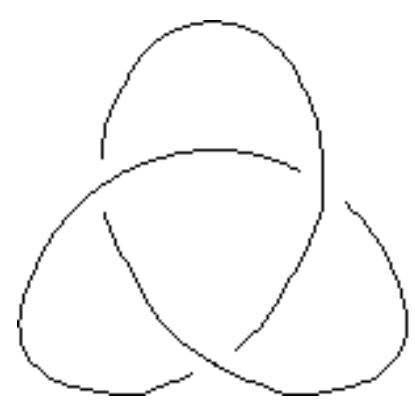

(a) trébol derecho

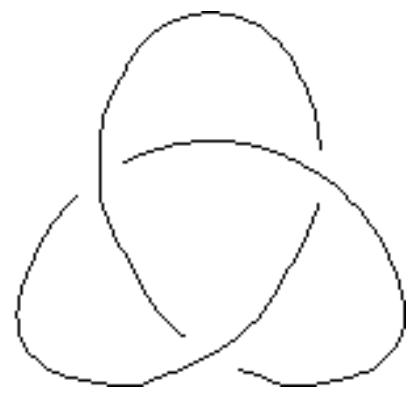

(b) trébol izquierdo

Figura 6. Dos tipos distintos de trébol. 
uno de nuestros nudos, la cual preservamos en el transcurso de la rotación, terminaremos con el reverso del nudo: la misma inmersión pero con la orientación opuesta. Por esta razón, decimos que los nudos trébol y doble son reversibles. No ocurre así en general, pues hay ejemplos de nudos, ya sean quirales o aquirales, que a la vez son irreversibles. ${ }^{6}$

\section{Nudos geométricos}

Consideremos las diferentes configuraciones que se pueden construir con una secuencia de segmentos lineales, unidos de punta a punta hasta formar un polígono inmerso en $\mathbb{R}^{3}$. Los segmentos representan ya sea enlaces entre átomos en un polímero, segmentos en la secuencia de pares de bases en una macromolécula de ADN circular, o simplemente palitos de madera unidos con articulaciones de hule flexible. Así un polígono espacial de este tipo sirve como modelo matemático para algún objeto que está físicamente anudado pero aún mantiene cierta cantidad de rigidez que hereda de los materiales de los cuales se construye.

Según un resultado clásico de la topología tridimensional, a todo nudo construido de cuerda flexible se le puede aproximar con una curva poligonal construida de segmentos delgados y rígidos. Además, cualquier deformación a la cual se someta la cuerda puede ser aproximada por una deformación del polígono, siempre y cuando se pueda aumentar el número de lados. Sin embargo, si se insiste en que el número de lados se mantenga constante, claramente se restringen los tipos de nudos que se pueden construir. Por ejemplo, cualquier polígono que podamos construir utilizando solamente cinco segmentos (o menos) representará topológicamente un nudo trivial; en cambio, podemos construir un nudo trébol o doble si utilizamos seis o siete segmentos, respectivamente. Ver la Figura 7. Lo que no es nada claro a primera vista es si aún se puede remedar una deformación topológica con una deformación de polígonos cuando se le ponen restricciones al número de segmentos. Por ejemplo, se desconoce si es posible construir un polígono lo suficientemente complicado para que al ser construido de cuerda flexible se pueda deformar topológicamente a un nudo trivial redondo, pero que al ser construido con segmentos rígidos y articulaciones flexibles no se pueda convertir en un simple polígono plano. En otras palabras, es todavía un problema abierto el encontrar nudos topológicos triviales que estén geométricamente atascados.

Resulta que a veces no existe ninguna isotopía geométrica (i.e. una que mantenga constante el número de segmentos) entre dos configuraciones poligonales correspondientes al mismo nudo. Es más, ni el caso de los tréboles hexagonales es trivial, ya que existen dos tipos geométricos distintos de este nudo. Como consecuencia, aún las propiedades familiares, como la reversibilidad, se comportan de manera diferente cuando se trata de nudos geométricos.

Una formulación útil debida a Dick Randell $[22,23]$ se obtiene al observar la correspondencia entre los polígonos de $n$ lados en el espacio euclídeo de tres dimensiones y ciertos puntos de $\mathbb{R}^{3 n}$. Supongamos que $P$ es un polígono de $n$ lados inmerso en $\mathbb{R}^{3}$, al cual se le ha escogido un "primer vértice" $v_{1}$ al igual que una orientación. Al escribir las coordenadas de cada vértice en un $3 n$-tuplo, obtenemos un punto $\left(x_{1}, y_{1}, z_{1}, x_{2}, y_{2}, z_{2}, \ldots, x_{n}, y_{n}, z_{n}\right) \in$ $\mathbb{R}^{3 n}$, el cual asociamos con $P=\left\langle v_{1}, v_{2}, \ldots, v_{n}\right\rangle$. Definimos el discriminante $\Sigma^{(n)}$ como el

6 Los nudos $8_{17}$ y $9_{32}$ son quirales e irreversibles. Sin embargo, el anverso y el reverso del nudo $8_{17}$ son equivalentes, mientras que $9_{32}$, su anverso, su reverso y su inverso (el reverso del anverso) no son equivalentes entre sí. 


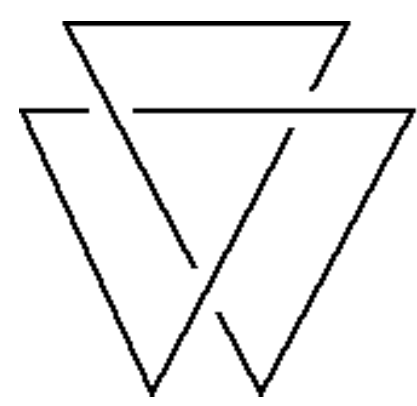

(a) trébol hexagonal

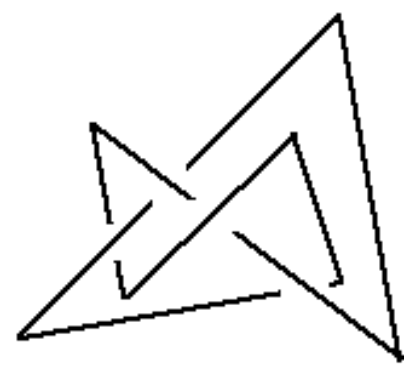

(b) doble heptagonal

FiguRA 7. Un nudo trébol hexagonal y un nudo doble heptagonal.

conjunto de puntos en $\mathbb{R}^{3 n}$ que corresponden a polígonos con auto-intersecciones. Si $n>3$, este discriminante es la unión de $\frac{1}{2} n(n-3)$ piezas, cada una de las cuales corresponde al conjunto de polígonos con intersección entre un par de lados no-adyacentes en particular. Por ejemplo, el subconjunto de $\Sigma^{(n)}$ que corresponde a polígonos con intersección entre los lados $v_{1} v_{2}$ y $v_{3} v_{4}$ se puede describir como la colección de polígonos para los cuales:

(i) los vértices $v_{1}, v_{2}, v_{3}$ y $v_{4}$ son coplanales,

(ii) la línea determinada por $v_{1}$ y $v_{2}$ separa a $v_{3}$ de $v_{4} \mathrm{y}$

(iii) la línea determinada por $v_{3}$ y $v_{4}$ separa a $v_{1}$ de $v_{2}$.

Nótese que este conjunto corresponde a la cerradura del lugar geométrico en $\mathbb{R}^{3 n}$ del sistema

$$
\begin{gathered}
\left(v_{2}-v_{1}\right) \times\left(v_{3}-v_{1}\right) \cdot\left(v_{4}-v_{1}\right)=0, \\
\left(v_{2}-v_{1}\right) \times\left(v_{3}-v_{1}\right) \cdot\left(v_{2}-v_{1}\right) \times\left(v_{4}-v_{1}\right)<0, \\
\left(v_{4}-v_{3}\right) \times\left(v_{1}-v_{3}\right) \cdot\left(v_{4}-v_{3}\right) \times\left(v_{2}-v_{3}\right)<0 .
\end{gathered}
$$

Por lo tanto, cada una de estas piezas es la cerradura de una variedad semialgebraica cúbica de codimensión uno, i.e. una hipersuperficie con frontera. Definimos que el espacio de nudos geométricos sea el complemento $\mathfrak{G} \mathfrak{e o}^{(n)}=\mathbb{R}^{3 n}-\Sigma^{(n)}$ de este discriminante. De esta manera, $\mathfrak{G}_{\mathfrak{e q}}{ }^{(n)}$ es una subvariedad topológica densa y abierta en $\mathbb{R}^{3 n}$ en la cual puntos corresponden a polígonos inmersos o nudos geométricos, arcos corresponden a isotopías geométricas y arco-componentes corresponden a tipos geométricos de nudos.

Por un teorema de Whitney, para cada $n$ hay solamente un número finito de arco-

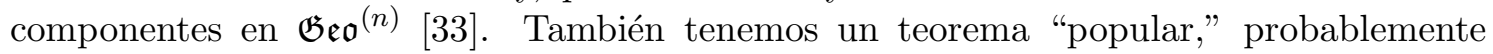
originario de Kuiper, el cual dice que los espacios $\mathfrak{G}_{\mathfrak{e o}}{ }^{(3)}, \mathfrak{G}_{\mathfrak{e o}}{ }^{(4)}$ y $\mathfrak{G}_{\mathfrak{e o}}{ }^{(5)}$ son arco-conexos. En $[4,5]$, se demuestra que cada uno de los espacios $\mathfrak{G e o}^{(6)}$ y $\mathfrak{G}_{\mathfrak{e o}}{ }^{(7)}$ contiene cinco componentes. En contraste a éste, tenemos el hecho que sólo hay tres tipos topológicos de

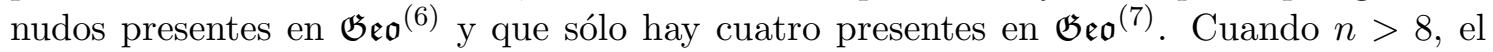
número exacto de arco-componentes todavía se desconoce. Es más, se conoce el número de tipos topológicos de nudos representados en los diversos componentes de $\mathfrak{G e o}^{(n)}$ solamente cuando $n<9$. El siguiente teorema resume el estado presente de la clasificación de los nudos geométricos con número pequeño de lados.

Teorema 1. (Calvo $[4,5])$ 
(i) Los espacios $\mathfrak{G e o}^{(3)}, \mathfrak{G} e \mathfrak{o}^{(4)}$ y $\mathfrak{G e} \mathfrak{o}^{(5)}$ son arco-conexos y constan solamente de nudos triviales.

(ii) El espacio $\mathfrak{G} \mathfrak{e o}^{(6)}$ de nudos hexagonales contiene cinco arco-componentes. Estos consisten en un solo componente de nudos triviales, dos componentes de tréboles derechos y dos componentes de tréboles izquierdos.

(iii) El espacio $\mathfrak{G}_{\mathfrak{e} \mathfrak{o}^{(7)}}$ de nudos heptagonales contiene cinco arco-componentes. Estos consisten en un solo componente de nudos triviales y de cada tipo topológico de nudo trébol y en dos componentes de nudos dobles.

(iv) El espacio $\mathfrak{G} \mathfrak{e o}^{(8)}$ de nudos octogonales contiene por lo menos veinte arco-componentes. Sin embargo, los únicos nudos presentes en este espacio son el nudo trivial, el nudo trébol, el nudo doble, todos los nudos primos de cinco y seis cruces $\left(5_{1}, 5_{2}, 6_{1}, 6_{2}\right.$ y $\left.6_{3}\right)$, el nudo "abuelita" y el nudo "cuadrado" $\left(3_{1} \pm 3_{1}\right)^{7}$ y los nudos de ocho cruces $8_{19}$ y $8_{20}$. Ver la Figura 8.

Es importante marcar que aunque las deformaciones que se obtienen como arcos en $\mathfrak{G}_{\mathfrak{e} \mathfrak{o}^{(n)}}$ preservan la estructura poligonal del nudo con el cual tratamos, en general éstas no preservan la longitud de sus segmentos. Sea $f: \mathfrak{G}_{\mathfrak{e o}}{ }^{(n)} \rightarrow \mathbb{R}^{n}$ el mapa que lleve el polígono $P=\left\langle v_{1}, v_{2}, \ldots, v_{n}\right\rangle$ al $n$-tuplo

$$
\left(\left\|v_{1}-v_{2}\right\|,\left\|v_{2}-v_{3}\right\|, \ldots,\left\|v_{n-1}-v_{n}\right\|,\left\|v_{n}-v_{1}\right\|\right) .
$$

Puntos en la preimagen $\mathfrak{E} \mathfrak{q u} \mathfrak{u}^{(n)}=f^{-1}(1,1, \ldots, 1)$ corresponden a nudos equiláteros con segmentos de longitud unitaria. Como el punto $(1,1, \ldots, 1)$ es un valor regular de $f$, el espacio $\mathfrak{E} \mathfrak{q u}{ }^{(n)}$ es una subvariedad suave de dimensión $2 n$ (en realidad, una hipersuperficie cuádrica de codimensión $n$ ) que intersecta varios de los componentes de $\mathfrak{G}_{\mathfrak{e}}{ }^{(n)}$, algunos posiblemente más de una sola vez. Arcos en esta subvariedad corresponden a isotopías geométricas que además preservan la longitud de los segmentos; por lo tanto los arcocomponentes de este espacio nos ofrecen otra noción más de anudamiento.

En sus escritos originales sobre los espacios de conformaciones moleculares, Randell demostró que si $n \leq 5$ entonces $\mathfrak{E q u}{ }^{(n)}$ es conexo [22, 23]. El caso $n=6$ no fue tocado por casi diez años, con excepción del trabajo de Ken Millett y Rosa Orellana quienes probaron que $\mathfrak{E q u}{ }^{(6)}$ contiene un solo componente de nudos triviales topológicos. ${ }^{8}$ Enfocando la atención a un caso especial de hexágonos singulares "casi anudados," se demuestra en [5] que un par de hexágonos son equivalentes equiláteramente si y sólo si lo son geométricamente. Así, $\mathfrak{E q u}{ }^{(6)}$ intersecta cada componente de $\mathfrak{G}_{\mathfrak{e}}{ }^{(6)}$ solamente una vez. Además, se prueba que esta correspondencia de arco-componentes no deja de ser interesante, pues la inclusión $\mathfrak{E q u}^{(6)} \hookrightarrow \mathfrak{G} \mathfrak{e o}^{(6)}$ tiene, a nivel de grupo fundamental, un núcleo no trivial. Es más, si $\mathcal{T}$ es un componente de nudos tréboles en ${\mathfrak{G} \mathfrak{e}{ }^{(6)}}^{(6)}$ entonces $\pi_{1}(\mathcal{T})=\mathbb{Z}_{2}$ mientras que $\pi_{1}\left(\mathcal{T} \cap \mathfrak{E q u}{ }^{(6)}\right)$ contiene un subgrupo cíclico infinito.

\section{Una estratificación del espacio de nudos geométricos}

7 El nudo abuelita se forma al amarrar dos nudos tréboles derechos, uno depués del otro. El nudo cuadrado se forma al atar un trébol derecho seguido por uno izquierdo. Por lo tanto la notación AlexanderBriggs les llama $3_{1}+3_{1}$ y $3_{1}-3_{1}$, respectivamente. La razón por sus curiosos nombres viene de la tradición marítima, la cual asegura que el nudo abuelita se afloja fácilmente y que el cuadrado da mejor sostén.

$8 \mathrm{Su}$ resultado nunca fue publicado, pero se menciona en la Preposición 1.2 de [20]. Una prueba alterna aparece en [5]. 


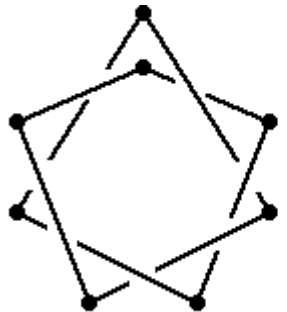

$5_{1}$

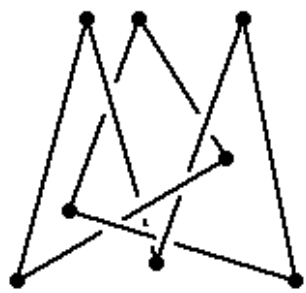

62

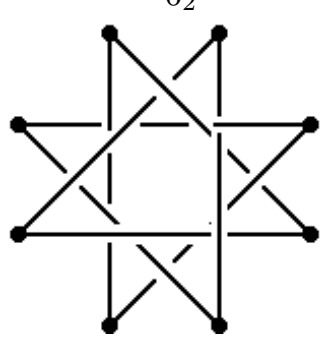

$3_{1}-3_{1}$

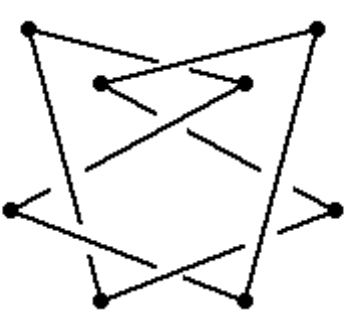

$5_{2}$

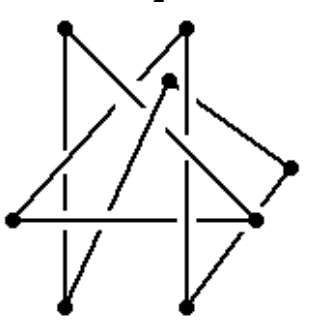

63

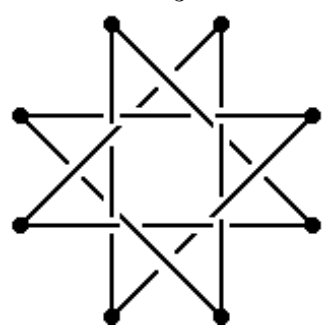

819
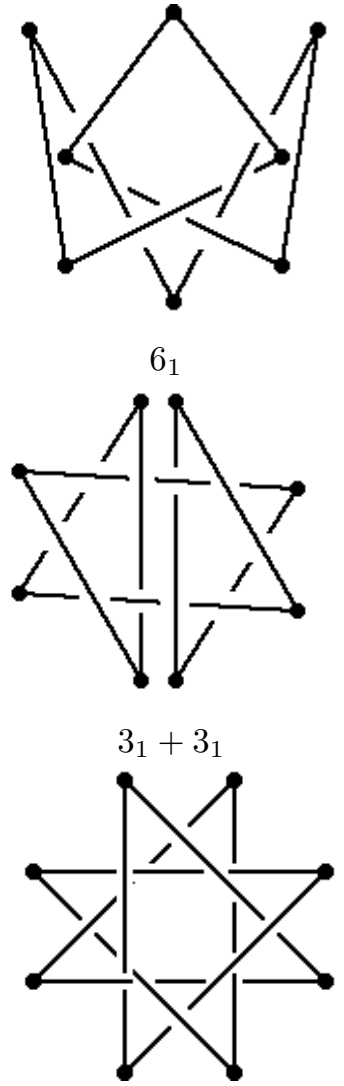

$8_{20}$

Figura 8. Todos los nudos octogonales.

Consideremos la función $g$ de dominio $\mathfrak{G} e \mathfrak{o}^{(n)}$ que se "olvida" del último vértice de un polígono, llevando

$$
P=\left\langle v_{1}, v_{2}, v_{3}, \ldots, v_{n-1}, v_{n}\right\rangle \mapsto g(P)=\left\langle v_{1}, v_{2}, v_{3}, \ldots, v_{n-1}\right\rangle .
$$

Un polígono genérico en $\mathfrak{G}_{\mathfrak{e o}}{ }^{(n)}$ tiene por imagen a un polígono inmerso de $\mathfrak{G}_{\mathfrak{e q}}{ }^{(n-1)}$; los únicos polígonos para los cuales esto no ocurre son aquéllos en que alguna parte de la cadena lineal a trozos $v_{1} v_{2} \ldots v_{n-1}$ pasa a través del segmento entre $v_{1}$ y $v_{n-1}$, y estos polígonos forman un subconjunto de codimensión uno en $\mathfrak{G e o}^{(n)}$. En particular, como ${\mathfrak{G} \mathfrak{e} \mathfrak{o}^{(n)}}^{(n)}$ una variedad topológica, cada polígono de $n$ segmentos se puede perturbar por una cantidad minúscula de manera que su imagen bajo $g$ se encuentre en $\mathfrak{G}_{\mathfrak{e o}}{ }^{(n-1)}$.

Supongamos que $Q$ es un polígono de $n-1$ segmentos en $\mathfrak{G}_{\mathfrak{e}}{ }^{(n-1)}$. Entonces la preimagen $g^{-1}(Q)$ será una variedad topológica tridimensional homeomórfica al conjunto de $n^{\text {avos }}$ vértices "válidos" para $Q$. Esto divide a $\mathfrak{G}_{\mathfrak{e o}}{ }^{(n)}$ en rebanadas o estratos tridimensionales. Así como $Q$ varía a través de $\mathfrak{G}_{\mathfrak{e} \mathfrak{o}^{(n-1)}}$, el estrato correspondiente a $g^{-1}(Q)$ también cambiará de aspecto. Al observar cómo cambian estos estratos, podremos obtener información valiosa sobre la estructura de $\mathfrak{G}_{\mathfrak{e}}{ }^{(n)}$. 
Por ejemplo, consideremos el pentágono $Q=\left\langle v_{1}, v_{2}, v_{3}, v_{4}, v_{5}\right\rangle$ con coordenadas

$$
\begin{gathered}
\langle(0,0,0),(.886375, .276357, .371441), \\
(.125043,-.363873, .473812), \\
(.549367, .461959, .845227),(.818041,0,0)\rangle
\end{gathered}
$$

ilustrado en la Figura 9. Podemos reemplazar el segmento entre $v_{5}$ y $v_{1}$ con un par de segmentos nuevos, desde $v_{5}$ hasta un nuevo vértice $v_{6} \in \mathbb{R}^{3}$ y de regreso a $v_{1}$. Esto creará un hexágono que, con un poquito de cuidado al escoger a $v_{6}$, también estará inmerso en $\mathbb{R}^{3}$. Si colocamos el nuevo vértice sobre el punto $(.4090205,0,-.912525)$, obtendremos un hexágono trivialmente anudado. En cambio, al poner a $v_{6}$ sobre $(.4090205,-.343939, .845227)$, obtenemos un hexágono que está anudado como un trébol derecho. Ver la Figura 9. La preimagen $g^{-1}(Q) \in{\mathfrak{G} e \mathfrak{o}^{(6)}}^{(6)}$ es homeomórfica al conjunto abierto y denso de $\mathbb{R}^{3}$ que consiste de aquellos puntos en $\mathbb{R}^{3}$ que se prestan como sextos vértices "válidos" para $Q$.

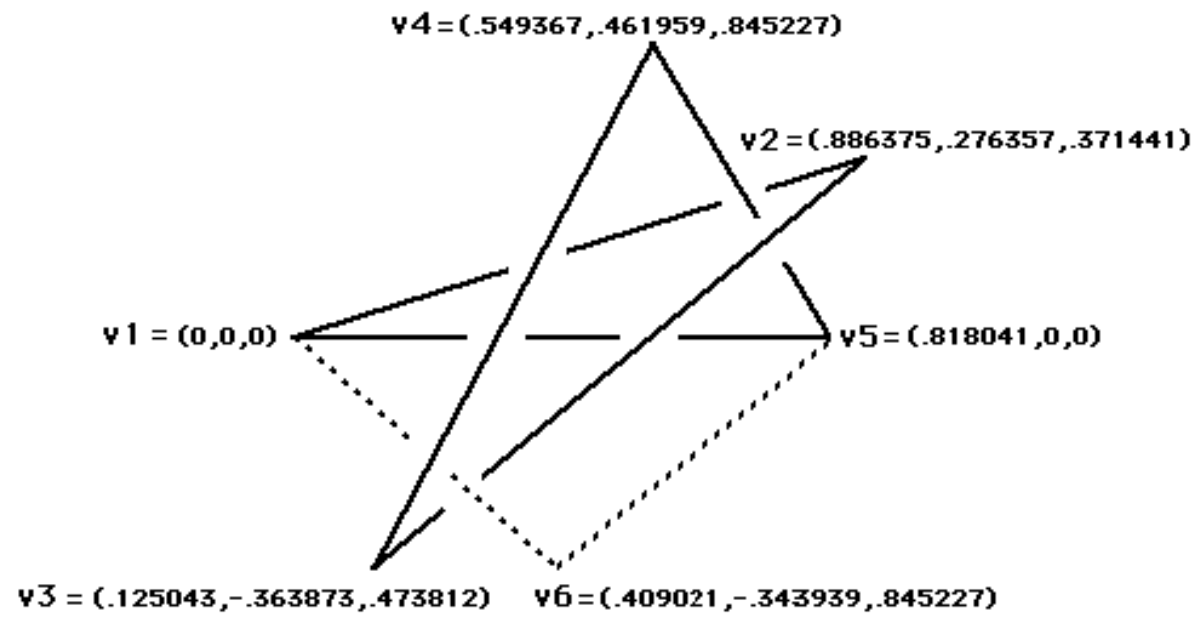

Figura 9. Una posible vista del pentágono $Q$.

Para examinar cuáles puntos en $\mathbb{R}^{3}$ corresponden a hexágonos inmersos procedentes de $Q$, tomamos el eje $x$ como un "eje central" en este espacio, mientras consideramos la colección de medios planos que radían de este eje. Nos referimos a éstos como medios planos estándar. Estos medios planos aparecen como rayos saliendo del origen en la Figura 10, que muestra la proyección de $Q$ al plano $y z$.

Sean $\mathcal{P}_{2}, \mathcal{P}_{3}$ y $\mathcal{P}_{4}$ los medios planos estándar que contienen a $v_{2}, v_{3}$ y $v_{4}$, respectivamente. O sea,

$$
\begin{gathered}
\mathcal{P}_{2}=\left\{y=\frac{276357}{371441} z \approx .744 z, z>0\right\}, \\
\mathcal{P}_{3}=\left\{y=-\frac{363873}{473812} z \approx-.768 z, z>0\right\}, \\
\mathcal{P}_{4}=\left\{y=\frac{461959}{845227} z \approx .547 z, z>0\right\},
\end{gathered}
$$

como se logra observar en la Figura 10.

Nótese que ningún medio plano estándar a la izquierda de $\mathcal{P}_{2}$ y $\mathcal{P}_{3}$ llega a tocar a $Q$ en su interior. Por eso, cualquier punto en el interior de cualquiera de estos medios planos se 


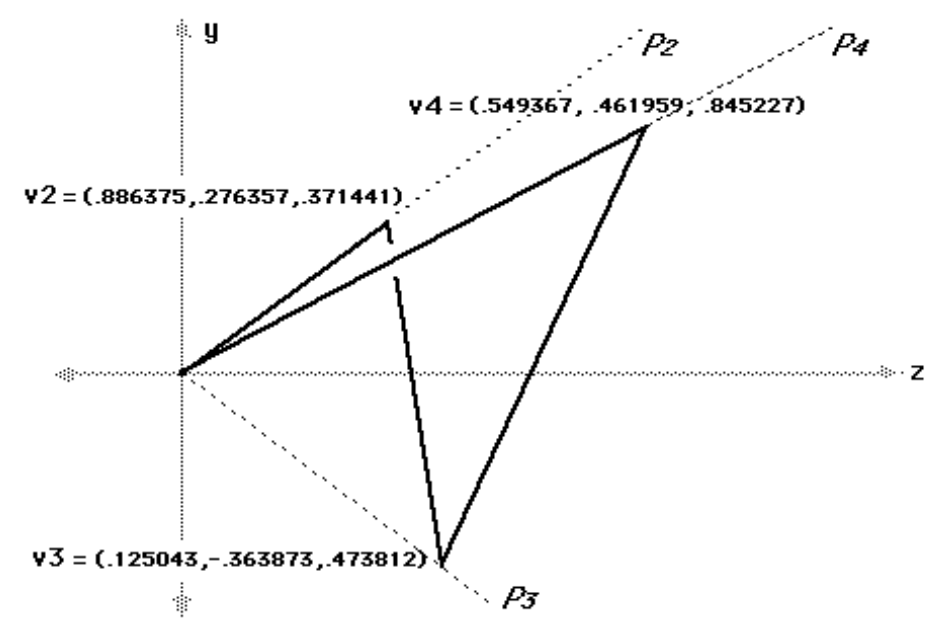

Figura 10. Proyección del pentágono $Q$ al plano yz.

puede usar como el sexto vértice de un hexágono. Sin embargo, los demás medios planos sí intersectan a $Q$ en por lo menos uno de sus puntos interiores, por lo que estos medios planos contendrán ciertos puntos que corresponden a hexágonos con auto-intersecciones.

El interior de un medio plano estándar entre $\mathcal{P}_{2}$ y $\mathcal{P}_{4}$ intersectará a $Q$ sólo una vez, en su segundo segmento. Dependiendo de cuál punto en este plano escojamos como el nuevo vértice $v_{6}$, la cadena de dos segmentos $v_{5} v_{6} v_{1}$ pasará o por debajo de este segmento o por encima de él. Si $v_{5} v_{6} v_{1}$ pasa por debajo de $v_{2} v_{3}$, entonces $v_{6}$ se puede empujar hacia el eje $x$, digamos que al punto medio del segmento $v_{1} v_{5}$, dándonos una isotopía desde nuestro nuevo hexágono hasta el nudo trivial realizado por el pentágono $Q$. Sin embargo, si $v_{5} v_{6} v_{1}$ pasa por encima del segmento $v_{2} v_{3}$, entonces este segmento obstruirá cualquier isotopía del hexágono que trate de empujar a $v_{6}$ hacia el eje $x$ en este medio plano. Por ejemplo, $Q$ cruza el medio plano $\{y=.6 z, z>0\}$ en el punto $(.828333, .227547, .379246)$. Vértices colineales con $(.828333, .227547, .379246)$ y $v_{1}$ corresponden a hexágonos inmersos sólo al estar ubicados entre estos dos puntos; de otra manera el segundo y el sexto segmento del nuevo hexágono se intersectarán. Similarmente, aquellos vértices colineales con $(.828333, .227547, .379246)$ y $v_{5}$ que no se encuentren entre estos dos puntos corresponden a hexágonos cuyo segundo y quinto segmento se intersectan. Por lo tanto, aquellos vértices en uno de los dos rayos que comienzan en $(.828333, .227547, .379246)$ y se alejan de $v_{1} \mathrm{y}$ $v_{5}$ no corresponden a hexágonos inmersos, y el medio plano se corta en dos regiones por un discriminante con forma de "V." Ver la Figura 11(a). Si ponemos a $v_{6}$ en la región $i$ de este medio plano, entonces el nuevo par de segmentos pasará por debajo del segmento $v_{2} v_{3}$ y obtendremos un hexágono isotópico a $Q$. Al contrario, si $v_{6}$ se ubica en la región $i i$, entonces $v_{5} v_{6} v_{1}$ pasará por encima de este segmento.

Ahora, el interior de cada medio plano estándar entre $\mathcal{P}_{4}$ y $\mathcal{P}_{3}$ intersecta a $Q$ en dos puntos, en el interior de su segundo y de su tercer segmento. Como occurió anteriormente, estos segmentos forman obstrucciones a las isotopías que mueven a $v_{6}$ dentro de este medio plano. Por lo tanto, para cada punto en el cual estos segmentos cruzan el medio plano, habrá un discriminante con forma de "V". Por ejemplo, en el medio plano $\{y=0, z>0\}$, 


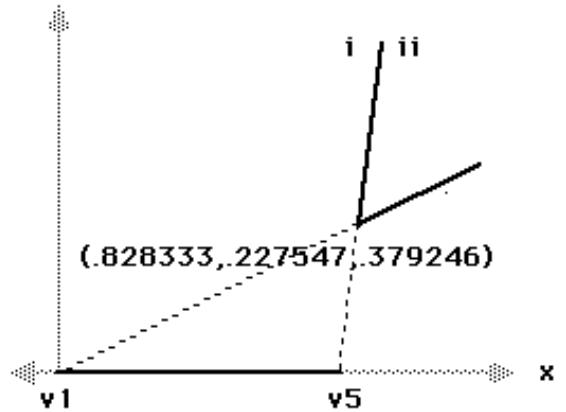

(a) $\{y=.6 z, z>0\}$

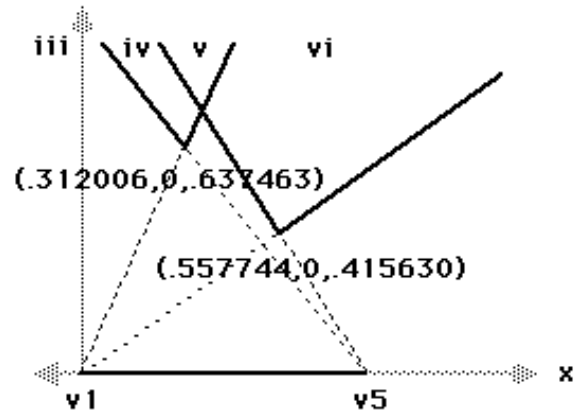

(b) $\{y=0, z>0\}$

Figura 11. Q separa a cada medio plano con discriminantes con forma de "V."

el cual intersecta a $Q$ en los puntos $(.557744,0, .415630)$ y $(.312006,0, .637463)$, todo vértice que forma parte de uno de los cuatro rayos que comienzan en uno de estos puntos y se alejan de $v_{1}$ y $v_{5}$ corresponde a un hexágono con auto-intersecciones. Estos dos discriminantes con forma de "V" separan al medio plano en cuatro regiones, como lo demuestra la Figura 11(b). Como antes, el colocar el nuevo vértice en alguna de estas regiones corresponde a enhebrar el par de nuevos segmentos del hexágono ya sea únicamente sobre el segundo (vi) o el tercer ( $i v)$ segmento de $Q$, sobre ambos $(v)$ o sobre ninguno de éstos (iii).

Podemos demostrar que los discriminantes con forma de "V" se mantienen prácticamente estáticos en los planos a través de cada uno de estos intervalos. Es más, los componentes conexos de los medios planos en la Figura 11 son solamente secciones transversales de ciertos "sectores cilíndricos" de $g^{-1}(Q)$ que se arroyan alrededor del eje $x$. Denotamos a estos sectores como $i$, ii, iii, $i v, v$ y vi, utilizando la misma numeración de la Figura 11. Además, llamamos $o$ al sector de $g^{-1}(Q)$ que consiste de aquellos medios planos que del todo no intersectan a $Q$ en su interior. Así, la manera en que estos sectores se unen entre sí depende del comportamiento de los discriminantes en los tres medios planos de "nivel crítico" $\mathcal{P}_{2}, \mathcal{P}_{3}$ y $\mathcal{P}_{4}$

El primero de estos medios planos, $\mathcal{P}_{2}$, contiene el primer segmento de $Q$, el cual conecta a $v_{1}=(0,0,0)$ con $v_{2}=(.886375, .276357, .371441)$. Los vértices en los rayos que comienzan en cualquier punto de este segmento y se alejan de $v_{6}$ corresponden a hexágonos cuyo primer y quinto segmento se intersectan. Así, para cada punto en este segmento hay un discriminante con forma de "V". La unión de estos discriminantes forma un discriminante bidimensional que corresponde a una obstrucción en el espacio $g^{-1}(Q)$. Ver la Figura 12. Sin embargo, esta obstrucción sólo bloquea parcialmente el paso hacia el sector $i$. Por lo tanto, ambos $i$ y $i$ se unen a $o$ en este medio plano.

Un discriminante bidimensional similar aparece en el medio plano $\mathcal{P}_{4}$. Este contiene el cuarto segmento de $Q$, el cual conecta a $v_{4}=(.549367, .461959, .845227)$ con $v_{5}=$ $(.818041,0,0)$. Los vértices colineales con el origen y cualquier punto $p$ en este segmento corresponden a hexágonos inmersos únicamente si se encuentran entre $(0,0,0)$ y $p$. Ver la Figura 13. Este discriminante completamente cierra la entrada al sector $v i$, y obstruye parte de $i$, $i i$ y $i i i$. Así, en este nivel, $i$ se une a $i i i$ y a $i v$, $i i$ se une a $v$ y $v i$ se termina abruptamente. 


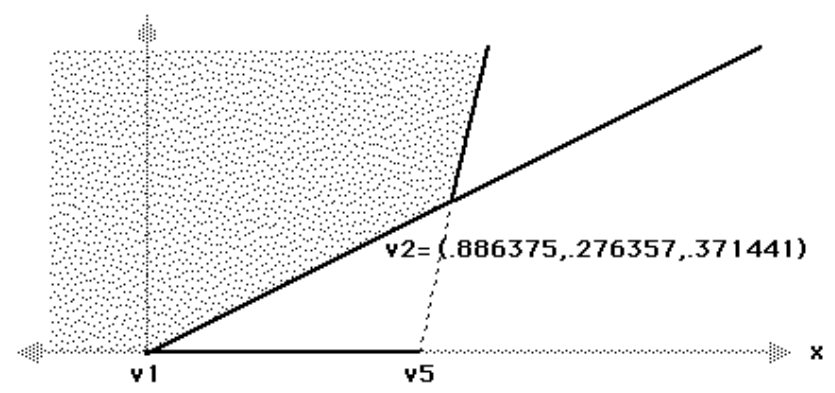

FigurA 12. Nivel crítico $\mathcal{P}_{2}=\left\{y=\frac{276357}{371441} z \approx .744 z, z>0\right\}$.

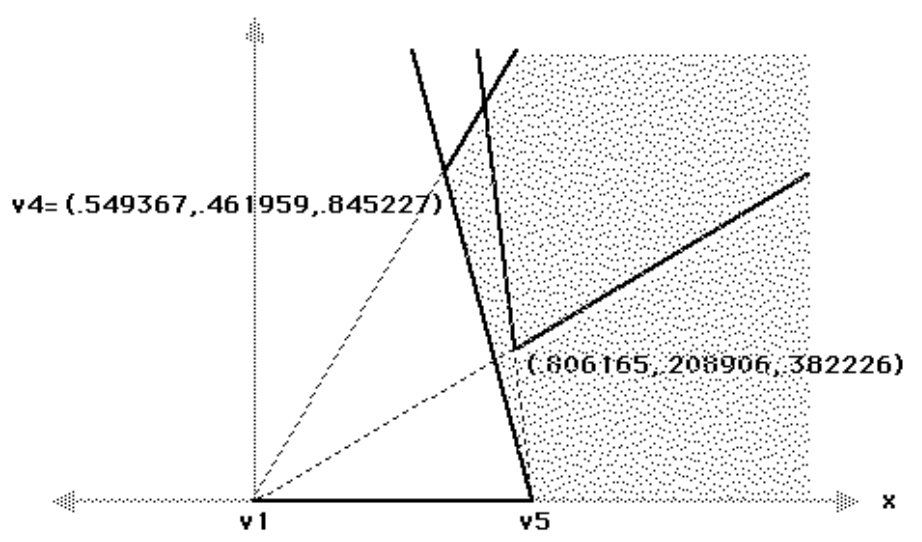

FigurA 13. Nivel crítico $\mathcal{P}_{4}=\left\{y=\frac{461959}{845227} z \approx .547 z, z>0\right\}$.

El medio plano al tercer nivel crítico, $\mathcal{P}_{3}$, nos presenta una situación diferente, pues éste intersecta a $Q$ sólo en el vértice $v_{3}=(.125043,-.363873, .473812)$. En este caso, los discriminantes con forma de "V" correspondientes al segundo y tercer segmento de $Q$ se unen cuando los dos segementos chocan en su vértice común. Al unirse estos discriminantes, los sectores $i v$ y $v i$ se cierran, mientras que los sectores $i i i$ y $v$ se unen al sector $o$.

La Figura 14 muestra una sección cilíndrica de $\mathbb{R}^{3}$ alrededor del eje $x$, revelando los sectores de $g^{-1}(Q)$ y las conecciones entre sí. En particular, se puede ver $\operatorname{como} g^{-1}(Q)$ consiste de dos arco-componentes disjuntos los cuales corresponden a los dos tipos de nudos que se pueden construir como hexágonos en el estrato $g^{-1}(Q)$ : el nudo trivial y el trébol.

La característica clave que determina el tipo de estrato asociado con un pentágono resulta ser la posición relativa del segundo, tercero y cuarto vértice con respecto al eje a través de los otros dos vértices. Supongamos que $Q=\left\langle v_{1}, v_{2}, v_{3}, v_{4}, v_{5}\right\rangle$ es un pentágono arbitrario en $\mathfrak{G}_{\mathfrak{e o}}{ }^{(5)}$ y que $\mathcal{L}$ es la línea determinada por $v_{1}$ y $v_{5}$. Como $\mathfrak{G}_{\mathfrak{e}}{ }^{(5)}$ es una variedad topológica, podemos modificar a $Q$ levemente, si es necesario, para asegurarnos de que $v_{1} v_{5}$ sea el único segmento de $Q$ que intersecta a $\mathcal{L}$. Como antes, sean $\mathcal{P}_{2}, \mathcal{P}_{3}$ y $\mathcal{P}_{4}$ los medios planos con frontera $\mathcal{L}$ que contienen a $v_{2}, v_{3}$ y $v_{4}$, respectivamente. De nuevo, 
TABla 1. Número de componentes en cada región de $\mathfrak{G e o}^{(6)}$.

\begin{tabular}{|cccc|}
\hline Región de $\mathfrak{G e o}^{(\mathbf{6})}$ & $\mathbf{0}$ & $\mathbf{3}_{\mathbf{1}}$ & $-\mathbf{3}_{\mathbf{1}}$ \\
\hline $2-3-4$ & 1 & - & - \\
$2-4-3$ & 1 & 1 & - \\
$3-2-4$ & 1 & 1 & - \\
$3-4-2$ & 1 & - & 1 \\
$4-2-3$ & 1 & - & 1 \\
$4-3-2$ & 1 & - & - \\
\hline
\end{tabular}

una pequeña deformación hace a $Q$ genérico, garantizando que los tres medios planos $\mathcal{P}_{i}$ sean distintos.

Como en el ejemplo previo, los $\mathcal{P}_{i}$ s dividen a $\mathbb{R}^{3}$ en tres regiones abiertas, de las cuales $Q$ intersecta a dos y completamente evita a la tercera. Si caminamos alrededor del eje $\mathcal{L}$ con un sentido "derecho," comenzando con la región que evita a $Q$, nos encontraremos con cada uno de los $\mathcal{P}_{i}$ s en alguno de los seis órdenes posibles. Por ejemplo, en el pentágono de las Figuras 9 y 10, estos medios planos aparecen en el orden $\mathcal{P}_{2}-\mathcal{P}_{4}-\mathcal{P}_{3}$, o simplemente 2-4-3.

Sea $H \in \mathfrak{G}_{\mathfrak{e q}}{ }^{(6)}$ un hexágono genérico inmerso en $\mathbb{R}^{3}$. Al considerar el orden en que aparecen los medios planos $\mathcal{P}_{i}$ asociados con $g(H)$, dividimos a $\mathfrak{G}_{\mathfrak{e}}{ }^{(6)}$ en seis regiones abiertas, las cuales se interconectan en conjuntos de codimensión uno donde ya sea:

(i) dos de $\operatorname{los} \mathcal{P}_{i}$ s coinciden o

(ii) un segmento de $H$ cruza a través de $\mathcal{L}$.

Al analizar los estratos $g^{-1}(g(H))$ y cómo se interconectan éstos, obtenemos la Tabla 1, la cual indica el número de arco-componentes en cada una de las seis regiones de $\mathfrak{G}_{\mathfrak{e} o}{ }^{(6)}$, organizados por el tipo topológico de nudo que éstos representan.

Como fue notado anteriormente, las seis regiones de $\mathfrak{G}_{\mathfrak{e o}}{ }^{(6)}$ fronterizan en conjuntos de codimensión uno consistiendo de hexágonos para los cuales coinciden dos de los $\mathcal{P}_{i}$ s. Por ejemplo, las regiones 2-4-3 y 4-2-3 se unen en un subconjunto que consiste de hexágonos

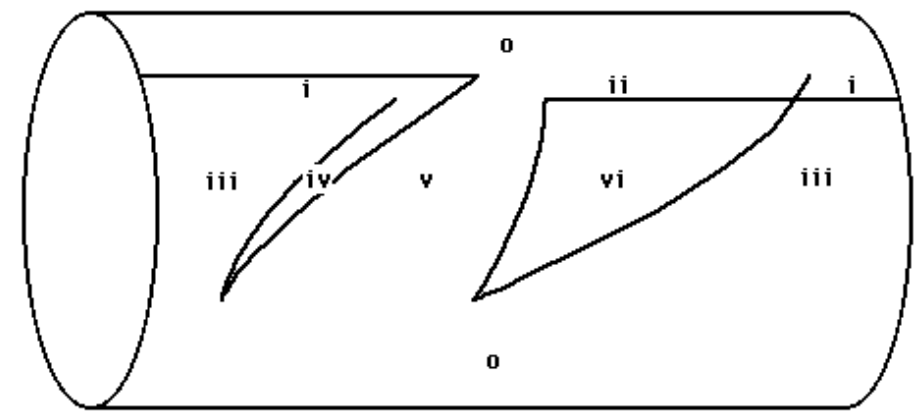

Figura 14. Una sección cilíndrica de $\mathbb{R}^{3}$ demostrando los diferentes sectores de $g^{-1}(Q)$ y sus interconecciones. 
con $\mathcal{P}_{2}=\mathcal{P}_{4}$. Las seis regiones también fronterizan en conjuntos de codimensión uno consistiendo de hexágonos que intersectan a la línea $\mathcal{L}$. Por ejemplo, las regiones 2-4-3 y 4-3-2 se unen en un conjunto de hexágonos para los cuales el segmento $v_{2} v_{3}$ intersecta esta línea. La Figura 15 demuestra todas estas conecciones; en esa figura, las líneas obscuras representan hexágonos con dos $\mathcal{P}_{i} \mathrm{~s}$ coincidentes mientras que las líneas grises representan hexágonos para los cuales algún segmento intersecta a la línea $v_{1} v_{5}$.

Consideremos un hexágono $H$ en la frontera común entre dos regiones de $\mathfrak{G} e \mathfrak{o}^{(6)}$. Como podemos modificar a $H$ levemente para obtener un hexágono genérico de cualquiera de los dos tipos, $H$ debe de ser de un tipo de nudo común a ambas regiones. Sin embargo, el único nudo común entre dos regiones adyacentes en la Figura 15 es el trivial. Por lo tanto, hexágonos en estos subconjuntos de codimensión uno deben de estar desanudados y, en particular, el conjunto de nudos triviales topológicos forma un solo componente de nudos triviales geométricos en $\mathfrak{G}_{\mathfrak{e o}}{ }^{(6)}$.

Al igual, supongamos que $h:[0,1] \rightarrow{\mathfrak{G} \mathfrak{e o}{ }^{(6)}}$ es un arco llevándonos de un nudo trébol en la región 2-4-3 a otro en la región 3-2-4. Como $\mathfrak{G e o}^{(6)}$ es un subconjunto abierto de $\mathbb{R}^{18}$, hay una pequeña bola de 18 dimensiones contenida en $\mathfrak{G e o}^{(6)}$ sobre cada punto en este arco. Por lo tanto, podemos asumir que cuando $h$ pasa a través de la frontera de una de las seis regiones, lo hace por un punto genérico en uno de los subconjuntos de codimensión uno descritos anteriormente. En este caso, $h$ tiene que pasar ya sea por la región 2-3-4 o 4-3-2; ver la Figura 15. Esta es una contradicción, ya que en estas regiones sólo viven nudos triviales. Así, no existe ningún arco que conecte los tréboles derechos de tipo 2-4-3 con los de tipo 3-2-4. Similarmente, no hay ningún arco entre los tréboles izquierdos de tipo 4-2-3 y los de tipo 3-4-2. Esto prueba que $\mathfrak{G}_{\mathfrak{e o}}{ }^{(6)}$ contiene cinco arco-componentes: uno que consiste de nudos triviales, dos de tréboles derechos, y dos de tréboles izquierdos.

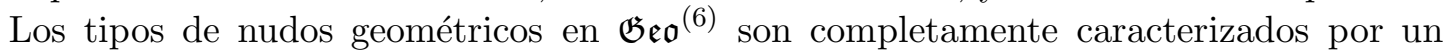
par de invariantes combinatorios que capturan la quiralidad topológica (i.e. derecha o izquierda) y la torsión geométrica (i.e. la "enroscadura" hacia arriba o abajo) de un hexágono, y que se calculan fácilmente por las coordenadas de sus vértices. Para definir estos invariantes, supongamos que $H=\left\langle v_{1}, v_{2}, v_{3}, v_{4}, v_{5}, v_{6}\right\rangle$ es un hexágono inmerso en $\mathbb{R}^{3}$ y consideremos el disco triangular abierto determinado por los vértices $v_{1}, v_{2}$ y $v_{3}$.

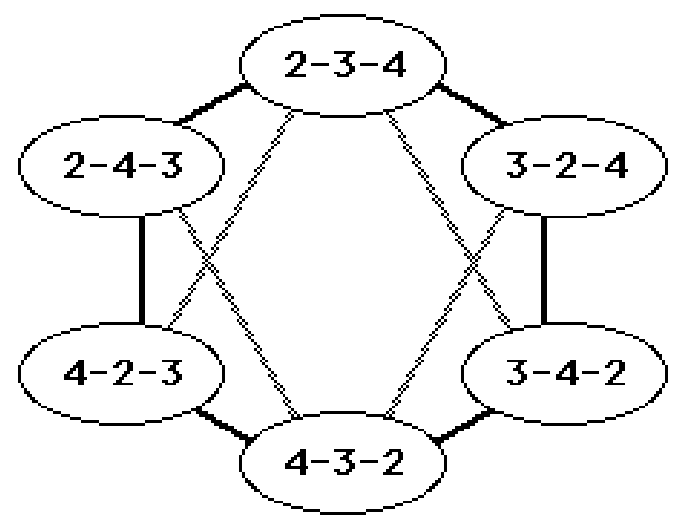

Figura 15. Conexiones de codimensión uno entre regiones. 
Este disco hereda una orientación de $H$ vía la "regla de la mano derecha." Sea $\Delta_{2}$ el número de intersección algebraico entre el hexágono y este triángulo. Nótese que el disco triangular puede ser penetrado solamente por los segmentos $v_{4} v_{5} \mathrm{y} v_{5} v_{6}$. Además, si estos dos segmentos intersectan al disco, lo hacen en direcciones opuestas, y sus contribuciones individuales a $\Delta_{2}$ se cancelan. Por lo tanto $\Delta_{2}$ toma un valor de 0,1 , o -1 . Al igual, definimos a $\Delta_{4}$ y $\Delta_{6}$ como los números de intersección de $H$ con los triángulos $\triangle v_{3} v_{4} v_{5}$ y $\triangle v_{5} v_{6} v_{1}$, respectivamente. Al considerar los valores posibles de los $\Delta_{i}$ s (ver Lema 8 en [5]), podemos demostrar que

(i) $H$ es un trébol derecho si y sólo si $\Delta_{2}=\Delta_{4}=\Delta_{6}=1$,

(ii) $H$ es un trébol izquierdo si y sólo si $\Delta_{2}=\Delta_{4}=\Delta_{6}=-1 \mathrm{y}$

(iii) $H$ es un nudo trivial si y sólo si $\Delta_{i}=0$ para algún $i \in\{2,4,6\}$, implicando que el producto

$$
\Delta(H)=\Delta_{2} \Delta_{4} \Delta_{6},
$$

al cual llamamos la quiralidad de $H$, es un invariante bajo deformaciones geométricas.

A continuación, definimos la torsión de $H$ como

$$
\text { tor } H=\operatorname{signo}\left(\left(v_{3}-v_{1}\right) \times\left(v_{5}-v_{1}\right) \cdot\left(v_{2}-v_{1}\right)\right) .
$$

Esto nos da el signo de la coordenada $z$ de $v_{2}$ cuando rotamos a $H$ hasta que $v_{1}, v_{3} \mathrm{y}$ $v_{5}$ se coloquen en el plano $x y$ en una manera positiva, y por lo tanto mide, en cierto sentido, si un hexágono se enrosca hacia arriba o hacia abajo. Consideremos un arco $h:[0,1] \rightarrow{\mathfrak{G} \mathfrak{e} \mathfrak{o}^{(6)}}^{(6)}$ cual le cambia la torsión de +1 a -1 a un nudo trébol hexagonal. En este caso, tiene que haber cierto punto en este arco para el cual el producto escalar triple en (2) es igual a cero. Este punto corresponde a un hexágono para el cual los vértices $v_{1}, v_{2}, v_{3}$ y $v_{5}$ son coplanares. Sin embargo, podemos probar que todo hexágono con esta propiedad corresponde a un nudo trivial, lo que nos da una contradicción. En particular, el producto $\Delta^{2}(H)$ tor $H$ también es un invariante bajo deformaciones geométricas. Un simple cálculo ahora prueba que todo trébol de tipo 2-4-3 o 4-2-3 tiene torsión positiva, mientras que todo trébol de tipo 3-2-4 or 3-4-2 tiene torsión negativa.

Teorema 2. Definimos la quiralidad-torsión de un hexágono $H$ como el par $\mathcal{J}(H)=$ $\left(\Delta(H), \Delta^{2}(H)\right.$ tor $\left.H\right)$. En este caso,

$$
\mathcal{J}(H)= \begin{cases}(0,0) & \text { si y sólo si } H \text { es un nudo trivial, } \\ (+1, t) & \text { si y sólo si } H \text { es un trébol derecho con tor } H=t, \\ (-1, t) & \text { si y sólo si } H \text { es un trébol izquierdo con tor } H=t .\end{cases}
$$

Por lo tanto, el tipo de nudo geométrico de un hexágono $H$ está completamente determinado por el valor de su quiralidad y su torsión.

Antes de dejar atrás el mundo de los hexágonos, hacemos una última observación. Recordamos que la construcción del espacio $\mathfrak{G}_{\mathfrak{e o}}{ }^{(n)}$ depende de la selección de un "primer vértice" $v_{1}$ y de una orientación. Esto viene a ser lo mismo que escoger una numeración $v_{1}, v_{2}, \ldots, v_{n}$ en secuencia para los vértices de cada polígono. Al escoger una numeración diferente llegamos a un punto diferente de $\mathfrak{G}_{\mathfrak{e}}{ }^{(n)}$ que corresponde al mismo polígono subyacente. Por lo tanto, el grupo diedral $\mathbf{D}_{n}$ de orden $2 n$ actúa sobre $\mathfrak{G}_{\mathfrak{e q}}{ }^{(n)}$ al incrementar o invertir esta numeración, y esta acción preserva el tipo de nudo topológico de un polígono. Sin embargo, si observamos los efectos que la acción de $\mathbf{D}_{6}$ sobre $\mathfrak{G}_{\mathfrak{e}}{ }^{(6)}$ tiene sobre $\mathfrak{t o r} H$, 
podemos ver que lo mismo no ocurre con el tipo de nudo geométrico de un polígono. En particular, si definimos la acción del grupo por los automorfismos

$$
\begin{aligned}
& r:\left\langle v_{1}, v_{2}, v_{3}, v_{4}, v_{5}, v_{6}\right\rangle \mapsto\left\langle v_{1}, v_{6}, v_{5}, v_{4}, v_{3}, v_{2}\right\rangle, \\
& s:\left\langle v_{1}, v_{2}, v_{3}, v_{4}, v_{5}, v_{6}\right\rangle \mapsto\left\langle v_{2}, v_{3}, v_{4}, v_{5}, v_{6}, v_{1}\right\rangle,
\end{aligned}
$$

entonces

$$
\mathfrak{t o r} r H=\mathfrak{t o r} s H=-\mathfrak{t o r} H .
$$

Esto demuestra que el nudo trébol hexagonal no es reversible: En contraste con los tréboles en la categoría topológica, al invertir la orientación de un trébol hexagonal obtenemos un nudo geométrico diferente. Además, el incrementar la numeración de los vértices también cambia el tipo de nudo geométrico de un trébol, así que, al tomar el cociente bajo esta acción, podemos ver que cada uno de los espacios de hexágonos orientados pero no basados

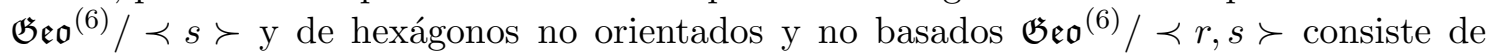
solamente tres arco-componentes.

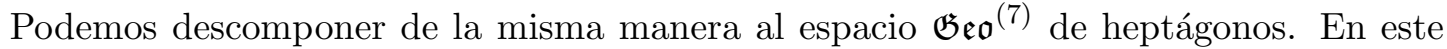
caso consideramos el orden relativo de los medios planos $\mathcal{P}_{2}, \mathcal{P}_{3}, \mathcal{P}_{4}$ y $\mathcal{P}_{5}$ cuya frontera es la línea determinada por $v_{1}$ y $v_{6}$. Esto define a 24 regiones abiertas de $\mathfrak{G}_{\mathfrak{e o}}{ }^{(7)}$, las cuales se interconectan en subconjuntos de codimensión uno en los cuales dos de los $\mathcal{P}_{i}$ s coinciden. Estas interconecciones se pueden describir esquemáticamente como intercambios en los índices que denotan a las regiones. Por ejemplo, las regiones 2-4-3-5 y 4-2-3-5 se conectan en un subconjunto consistiendo de heptágonos en los cuales $\mathcal{P}_{2}$ y $\mathcal{P}_{4}$ cambian de posición. Podemos construir un modelo de estas interconecciones si tomamos un vértice para cada una de las 24 regiones y un segmento para cada una de las conecciones de codimensión 1 entre ellas. El resultado es un grafo que forma el esqueleto de dimensión uno de un zonótopo sólido llamado permutaedro, presentado en la Figura 16. Cada vértice del permutaedro forma parte de una sola cara cuadrada que corresponde a la secuencia con orden 4 en la cual se intercambian, de manera alterna, los primeros dos y los últimos dos índices en nuestra notación. Además, cada vértice forma parte de dos diferentes caras hexagonales, las cuales corresponden a las secuencias con orden 6 en las cuales se fija ya sea el primer o el último índice mientras que los otros tres índices se permutan a través de los seis órdenes posibles. Por lo tanto el permutaedro de valencia 3 tiene sies caras cuadradas y ocho caras hexagonales. Al extender los segmentos comunes entre cada par de caras hexagonales adyacentes, notamos que el permutaedro no es más que un octaedro truncado, también conocido en la cristalografía como un cubo-octaedro de Fedorov. ${ }^{9}$

Después de ciertas complicaciones adicionales,${ }^{10}$ el análisis de los estratos en cada una de estas regiones demuestra que $\mathfrak{G}_{\mathfrak{e}}{ }^{(7)}$ contiene un solo arco-componente de nudos triviales y de cada tipo topológico de trébol, y dos que contienen nudos dobles. Estos nudos dobles son nuevos ejemplos de isótopos geométricos distintos del mismo nudo topológico, los cuales se distinguen gracias a un invariante geométrico $\Xi$, que definimos a continuación.

9 El cubo-octaedro es un paraleloedro, o sea, una figura cristalina con caras opuestas paralelas con el cual se puede teselar el espacio tridimensional. No se debe confundir el cubo-octaedro de Fedorov con el cuboctaedro de Kepler, el cual se obtiene de un octaedro al truncarlo en el punto medio (no en los puntos de un tercio y dos tercios) de cada segmento y por lo tanto consta de seis caras cuadradas y ocho caras triangulares. Ver pp. $17-18$ en [34] y pp. $722-723$ en [31].

10 Se dirige el lector interesado a ver pp. $53-56$ en [4]. 

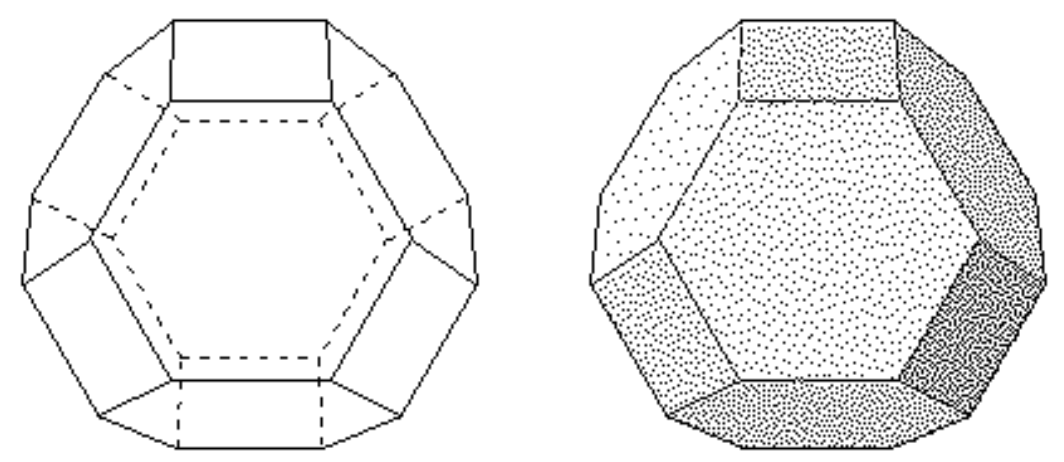

Figura 16. El permutaedro de valencia 3.

Supongamos que $H$ es el heptágono con vértices $\left\langle v_{1}, v_{2}, v_{3}, v_{4}, v_{5}, v_{6}, v_{7}\right\rangle$. Definimos las funciones $\Theta_{3}(H)$ y $\Theta_{6}(H)$ como los signos de los siguientes productos triples:

$$
\begin{aligned}
& \Theta_{3}=\operatorname{signo}\left(\left(v_{7}-v_{1}\right) \times\left(v_{2}-v_{1}\right) \cdot\left(v_{3}-v_{1}\right)\right), \\
& \Theta_{6}=\operatorname{signo}\left(\left(v_{6}-v_{1}\right) \times\left(v_{7}-v_{1}\right) \cdot\left(v_{2}-v_{1}\right)\right) .
\end{aligned}
$$

Entonces $\Theta_{3}=\Theta_{6}$ si los vértices $v_{3}$ y $v_{6}$ se encuentran en el mismo lado del plano $\mathcal{P}$ determinado por $v_{7}, v_{1}$ y $v_{2}$, mientras que $\Theta_{3}=-\Theta_{6}$ si $v_{3}$ y $v_{6}$ se encuentran en lados opuestos de $\mathcal{P}$. Notamos que para un heptágono genérico, exactamente una de las funciones $\frac{1}{2}\left(\Theta_{3}+\Theta_{6}\right)$ y $\frac{1}{2}\left(\Theta_{3}-\Theta_{6}\right)$ será igual a cero, mientras que la otra será \pm 1 .

Supongamos que $I_{34}$ denota el número de intersección algebraico entre el segmento $v_{3} v_{4}$ y el disco triangular $\triangle v_{7} v_{1} v_{2}$, utilizando las orientaciones usuales inducidas por $H$. Al igual, definimos a $I_{45}$ y a $I_{56}$ como los números de intersección entre el triángulo $\triangle v_{7} v_{1} v_{2}$ y los segmentos $v_{4} v_{5}$ y $v_{5} v_{6}$, respectivamente.

Si $H$ tiene $\Theta_{3}=\Theta_{6}$, entonces $v_{3}$ y $v_{6}$ están ubicados en el mismo lado del plano $\mathcal{P}$, o sea que la cadena lineal a trozos $v_{3} v_{4} v_{5} v_{6}$ intersecta a $\mathcal{P}$ no más de dos veces. Por supuesto, si ambas intersecciones ocurren en el interior de $\triangle v_{7} v_{1} v_{2}$, éstas tendrán orientaciones opuestas. Por lo tanto la suma $I_{34}+I_{45}+I_{56}$ toma solamente los valores -1, 0 o 1 .

Ahora, supongamos que $H$ es un nudo doble con $\Theta_{3}=-\Theta_{6}$. Entonces $v_{3}$ y $v_{6}$ se encuentran en lados opuestos del plano $\mathcal{P}$, de manera que la cadena lineal a trozos $v_{3} v_{4} v_{5} v_{6}$ intersecta a $\mathcal{P}$ un número impar de veces. Primero supongamos que sólo hay una intersección; entonces la cadena $v_{7} v_{1} v_{2}$ se puede isotopar linealmente a trozos en un segmento recto. Podemos visualizar esta isotopía como una deformación ya sea que empuja a $v_{1}$ en línea recta hacia el punto medio del segmento $v_{2} v_{7}$, o (en caso de que la intersección ocurra dentro del triángulo $\triangle v_{7} v_{1} v_{2}$ ) que dilata a $v_{7} v_{1} v_{2}$ en un lazo grande, el cual se gira alrededor del heptágono como quien "brinca suiza" y luego se contrae hasta coincidir con el segmento $v_{2} v_{7}$. Ver la Figura 17. En cualquier caso, obtendremos una realización hexagonal de un nudo doble. Como esto es imposible, la cadena $v_{3} v_{4} v_{5} v_{6}$ tiene que cruzar el plano $\mathcal{P}$ exactamente tres veces y, en particular, $v_{3} v_{4}$ y $v_{5} v_{6}$ lo intersectan con la misma orientación. Por lo tanto la cantidad $I_{34}-I_{56}$ será o cero (cuando los dos lados intersectan el interior de $\triangle v_{7} v_{1} v_{2}$, o cuando ninguno de los dos lo hace) o \pm 1 (cuando sólo una de estas intersecciones ocurre dentro del triángulo). 

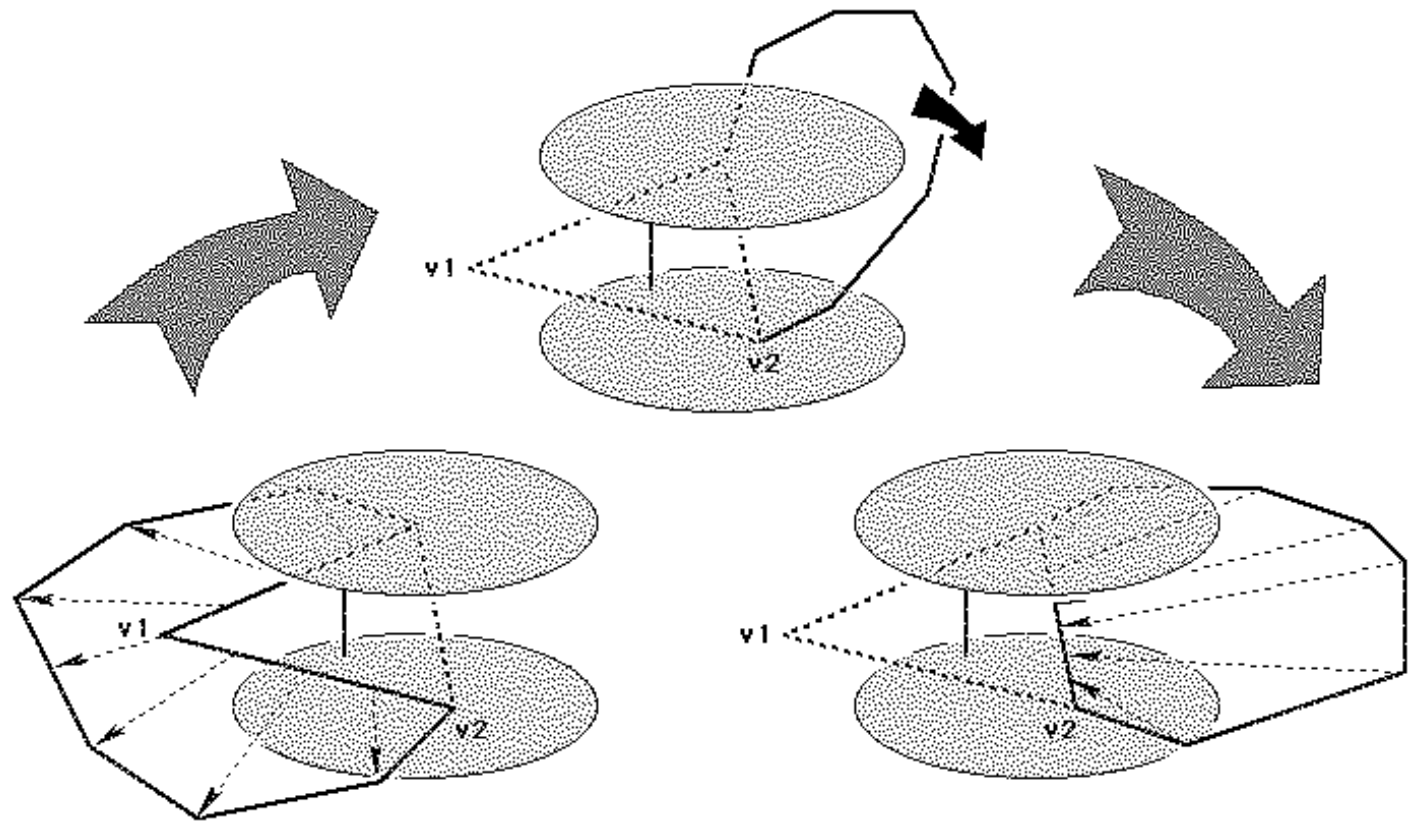

FiguRA 17. Isotopía lineal a trozos de la cadena $v_{7} v_{1} v_{2}$.

Si le echamos un vistazo a las configuraciones posibles, notamos que:

(i) si $H$ es un nudo doble heptagonal con $\Theta_{3}=\Theta_{6}$, entonces exactamente uno de los números de intersección $I_{34}, I_{45}$ o $I_{56}$ no será cero; por lo tanto $I_{34}+I_{45}+I_{56}= \pm 1$ (Lemma 4.2 in [4]) $\mathrm{y}$

(ii) si $H$ es un nudo doble heptagonal con $\Theta_{3}=-\Theta_{6}$, entonces exactamente uno de los números de intersección $I_{34}$ o $I_{56}$ no será cero; en particular $I_{34}-I_{56}= \pm 1$ (Lemma 4.3 in [4]).

Consideremos, entonces, a la función

$$
\Xi(H)=\frac{1}{2}\left(\Theta_{3}+\Theta_{6}\right)\left(I_{34}+I_{45}+I_{56}\right)+\frac{1}{2}\left(\Theta_{3}-\Theta_{6}\right)\left(I_{34}-I_{56}\right) .
$$

Como consecuencia de (i) y (ii), $\Xi$ toma solamente los valores de 1 o -1 . Supongamos que el valor de $\Xi$ cambia a través de un arco $h:[0,1] \rightarrow \mathfrak{G e o}^{(7)}$. Como $\mathfrak{G e o}^{(7)}$ es una variedad, podemos asumir que, en esta deformación, sólo un vértice de $H$ pasa por el interior de $\triangle v_{7} v_{1} v_{2}$ a la vez, que sólo un segmento intersecta a $v_{7} v_{2}$ a la vez y que estas dos cosas nunca ocurren al mismo tiempo. Nótese que cada uno de estos eventos cambiará los valores de $I_{34}+I_{45}+I_{56}$ y de $I_{34}-I_{56}$ por no más que uno. Sin embargo, $\Xi$ sólo cambia en incrementos de dos, así que si los valores de $\Theta_{3}$ y $\Theta_{6}$ se mantienen constantes a través de $h$, entonces $\Xi$ también se mantendrá constante.

Si es necesario, invertimos la orientación de $H$ para asumir que la deformación que corresponde al arco $h$ le cambia el signo a $\Theta_{3}$. En particular, sea $H_{0}$ un nudo doble heptagonal en este arco con $\Theta_{3}=0$. Al empujar a $v_{3}$ levemente hacia $v_{6}$, obtenemos un heptágono $H_{0}^{+}$con $\Theta_{3}=\Theta_{6}$; sea $I_{34}^{+}$el número de intersección apropiado para este heptágono. De la misma manera, al alejar a $v_{3}$ de $v_{6}$ obtendremos un heptágono $H_{0}^{-}$ 
con $\Theta_{3}=-\Theta_{6}$; sea $I_{34}^{-}$el número de intersección correspondiente a este heptágono. Al escoger a $H_{0}^{+}$y a $H_{0}^{-}$lo suficientemente cerca de $H_{0}$, podemos asumir que los valores de los números de intersección $I_{45}$ y $I_{56}$ coinciden para estos tres nudos. Esto nos deja dos casos que considerar.

En primer lugar, supongamos que $I_{34}^{-}=0$. Entonces $I_{56}= \pm 1$ por (ii), y por lo tanto $I_{34}^{+}=I_{45}=0$ por (i). En este caso,

$$
\left(I_{34}^{+}+I_{45}+I_{56}\right)=I_{56}=-\left(I_{34}^{-}-I_{56}\right) .
$$

El signo negativo en el término derecho de esta ecuación neutraliza el cambio de signo en $\Theta_{3}$, y así el valor de $\Xi$ permanece estático.

En segundo lugar, supongamos que $I_{34}^{-}= \pm 1$, en cuyo caso $I_{34}^{+}=0$. Entonces $I_{56}=0$ por (ii) y $I_{45}= \pm 1$ por (i). Además, los segmentos $v_{3} v_{4}$ y $v_{4} v_{5}$ de $H_{0}^{-}$intersectan al triángulo $\triangle v_{7} v_{1} v_{2}$ con direcciones opuestas, así que $I_{34}^{-}=-I_{45}$. Por lo tanto,

$$
\left(I_{34}^{+}+I_{45}+I_{56}\right)=I_{45}=-I_{34}^{-}=-\left(I_{34}^{-}-I_{56}\right)
$$

y, como antes, el valor de $\Xi$ no cambia. Esto prueba el siguiente resultado.

Teorema 3. $\Xi$ es un invariante de nudos dobles heptagonales bajo deformaciones geométricas.

Resulta interesante notar que $\Xi$ también es un invariante bajo anversos, ya que los cambios de signo que resultan en cada una de las funciones $\Theta_{3}, \Theta_{6}, I_{34}, I_{45}$ y $I_{56}$ se cancelan en (5). Esto refleja el hecho que el nudo doble heptagonal es aquiral, i.e. equivalente a su anverso. La Figura 18 demuestra una tal isotopía. Comenzando con el diagrama en la parte superior de la Figura 18 y procediendo hacia la derecha, primero empujamos a $v_{1}$ a través del interior del disco triangular $\triangle v_{2} v_{3} v_{4}$. Notamos que al hacer esto, es posible que necesitemos cambiar las longitudes de por lo menos uno de los segmentos del heptágono. Aunque resulta difícil verlo desde la perspectiva de la Figura 18, este movimiento en realidad define una isotopía desde el heptágono $\left\langle v_{1}, v_{2}, v_{3}, v_{4}, v_{5}, v_{6}, v_{7}\right\rangle$ hasta el heptágono $\left\langle-v_{6},-v_{7},-v_{1},-v_{2},-v_{3},-v_{4},-v_{5}\right\rangle$. Continuamos repitiendo una secuencia de movimientos semejantes, pasando a $v_{3}$ a través de $\triangle v_{4} v_{5} v_{6}$, luego a $v_{5}$ a través de $\triangle v_{6} v_{7} v_{1}$, etc. Después de siete pasos, cuando movemos a $v_{6}$ por el medio de $\triangle v_{7} v_{1} v_{2}$, llegamos al diagrama en la parte inferior de la Figura 18. Al llegar a este punto, el nudo doble se ha convertido en el anverso del original.

Finalmente, consideramos la acción del grupo diedral $\mathbf{D}_{7}$ sobre $\mathfrak{G}_{\mathfrak{e}}{ }^{(7)}$ definida por los automorfismos

$$
\begin{aligned}
& r\left\langle v_{1}, v_{2}, v_{3}, v_{4}, v_{5}, v_{6}, v_{7}\right\rangle=\left\langle v_{1}, v_{7}, v_{6}, v_{5}, v_{4}, v_{3}, v_{2}\right\rangle \\
& s\left\langle v_{1}, v_{2}, v_{3}, v_{4}, v_{5}, v_{6}, v_{7}\right\rangle=\left\langle v_{2}, v_{3}, v_{4}, v_{5}, v_{6}, v_{7}, v_{1}\right\rangle .
\end{aligned}
$$

Al invertir la orientación de $H$ vía el mapa $r$, se invierten las orientaciones de los segmentos de $H$ al igual que las de los discos triangulares definidos por éstos. En particular,

$$
I_{34}(r H)=I_{56}(H) \quad I_{45}(r H)=I_{45}(H) \quad I_{56}(r H)=I_{34}(H) .
$$


Sin embargo, $r$ no sólo intercambia las partes de $\Theta_{3}$ y de $\Theta_{6}$, sino también cambia sus signos:

$$
\begin{aligned}
\Theta_{3}(r H) & =\operatorname{signo}\left(\left(v_{2}-v_{1}\right) \times\left(v_{7}-v_{1}\right) \cdot\left(v_{6}-v_{1}\right)\right), \\
& =-\operatorname{signo}\left(\left(v_{6}-v_{1}\right) \times\left(v_{7}-v_{1}\right) \cdot\left(v_{2}-v_{1}\right)\right), \\
& =-\Theta_{6}(H), \\
\Theta_{6}(r H) & =\operatorname{signo}\left(\left(v_{3}-v_{1}\right) \times\left(v_{2}-v_{1}\right) \cdot\left(v_{7}-v_{1}\right)\right), \\
& =-\operatorname{signo}\left(\left(v_{7}-v_{1}\right) \times\left(v_{2}-v_{1}\right) \cdot\left(v_{3}-v_{1}\right)\right), \\
& =-\Theta_{3}(H) .
\end{aligned}
$$

Por lo tanto

$$
\begin{aligned}
\Xi(r H)= & \frac{1}{2}\left(\Theta_{3}(r H)+\Theta_{6}(r H)\right)\left(I_{34}(r H)+I_{45}(r H)+I_{56}(r H)\right) \\
& +\frac{1}{2}\left(\Theta_{3}(r H)-\Theta_{6}(r H)\right)\left(I_{34}(r H)-I_{56}(r H)\right) \\
= & \frac{1}{2}\left(-\Theta_{6}(H)-\Theta_{3}(H)\right)\left(I_{56}(H)+I_{45}(H)+I_{34}(H)\right) \\
& +\frac{1}{2}\left(-\Theta_{6}(H)+\Theta_{3}(H)\right)\left(I_{56}(H)-I_{34}(H)\right) \\
= & -\Xi(H) .
\end{aligned}
$$

Esto demuestra que, como los nudos tréboles hexagonales, los nudos dobles en $\mathfrak{G}_{\mathfrak{e o}}{ }^{(7)}$ son irreversibles, en contraste a sus contrapartes topológicas. Sin embargo, recordemos que la irreversibilidad de los tréboles en $\mathfrak{G}_{\mathfrak{e}}{ }^{(6)}$ se debe en gran parte a nuestra selección de un "primer" vértice $v_{1}$. En aquel caso, una permutación cíclica de los seis vértices cambiaría el tipo de nudo geométrico del trébol. Este no es el caso con los nudos dobles en $\mathfrak{G e o}^{(7)}$, pues consideremos la acción del grupo generado por el automorfismo $s$ sobre el conjunto de isótopos geométricos del nudo doble. Esta es una acción de orden 7 sobre un conjunto de dos elementos y por lo tanto tiene que ser trivial. O sea,

$$
\Xi(s H)=\Xi(H) \text {. }
$$

Así la distinción entre los dos tipos de nudos dobles es un efecto del "verdadero" anudamiento geométrico de los heptágonos, el cual llega más allá que una simple renumeración de los vértices o de nuestra selección arbitraria del primer vértice.

\section{Proyecciones de nudos y el índice poligonal mínimo}

La Sección 4 se dedica al problema de determinar, para un entero $n$, el número de arcocomponentes presentes en el espacio $\mathfrak{G e o}^{(n)}$ de polígonos inmersos con $n$ segmentos. En otras palabras, “¿Cuántos tipos de nudos geométricos hay para un valor particular de $n$ ?" $\mathrm{Al}$ crecer $n$, el espacio $\mathfrak{G} \mathfrak{e o}^{(n)}$ se hace combinatóricamente más y más intrincado. Cuando ésto ocurre, volvemos al problema de entender el número de tipos de nudos topológicos (en lugar de geométricos) que se presentan en $\mathfrak{G}_{\mathfrak{e o}}{ }^{(n)}$, y en particular preguntamos, "¿Qué tan 
complicado puede ser el nudo realizado por un polígono de $n$ segmentos?" La respuesta a esta pregunta se sabe únicamente cuando $n \leq 8$. Por ejemplo, sabemos que hay inmersiones poligonales de nueve segmentos para todo nudo primo de siete cruces $\left(7_{1}, \ldots, 7_{7}\right)$ al igual que para los nudos $8_{16}, 8_{17}, 8_{18}, 8_{21}, 9_{40}, 9_{41}, 9_{42}$ y $9_{46}$, pero se presume que esta lista puede ser mucho más grande e incluir varios de los nudos para los cuales hasta ahora sólo se ha encontrado realizaciones de diez u once segmentos. ${ }^{11}$ En esta sección, damos una cota superior a la complejidad de un nudo de $n$ segmentos.

Recordamos que el número mínimo de cruces de un nudo es el número más pequeño de cruces presente en cualquier proyección en posición general del nudo a un plano o una esfera. Esta es la medida convencional de la complejidad de un nudo, utilizada en la notación Alexander-Briggs y en las tablas canónicas de nudos primos. De manera similar, definimos el índice poligonal mínimo de un nudo como el número más pequeño de segmentos presentes en cualquier inmersión poligonal del nudo. ${ }^{12}$ Este invariante sirve como la medida correspondiente para la complejidad de los nudos poligonales. Tradicionalmente, la relación entre estos dos invariantes procede de la siguiente construcción. ${ }^{13}$

Sea $P=\left\langle v_{1}, v_{2}, \ldots, v_{n-1}, v_{n}\right\rangle \in \mathfrak{G e o}^{(n)}$ un polígono de $n$ segmentos inmerso en $\mathbb{R}^{3}$. Proyectamos los puntos de $P$ ortogonalmente a un plano perpendicular a alguno de los segmentos de $P$, digamos que a $v_{1} v_{2}$. Esto viene a ser lo mismo que mirar al polígono de manera que el segmento $v_{1} v_{2}$ se vea "de punta" y que la imagen de $P$ en nuestra retina (i.e. el plano) sea un polígono con $n-1$ segmentos. Ningún segmento en esta imagen puede cruzar ni a ninguno de sus vecinos ni a sí mismo y por lo tanto intersecta a un máximo de $n-4$ segmentos. De esta manera, cualquier polígono genérico de $\mathfrak{G}_{\mathfrak{e} o}{ }^{(n)}$ produce una proyección con un máximo de $\frac{1}{2}(n-1)(n-4)$ cruces. Esto nos lleva a la conclusión de que, si un nudo $K$ tiene un número mínimo de cruces $c(K)$ y un índice poligonal mínimo $p(K)$, entonces

$$
c(K) \leq \frac{(p(K)-1)(p(K)-4)}{2},
$$

o equivalentemente (al completar el cuadrado y resolver por $p$ )

$$
p(K) \geq \frac{5+\sqrt{9+8 c(K)}}{2} .
$$

Para los hexágonos y heptágonos, esta cota sobre el número de cruces es 5 y 9 , respectivamente, bastante pasada de los valores verdaderos de 3 y 4 que obtuvimos en la Sección 4. Es más, la cifra de $\frac{1}{2}(n-1)(n-4)$ cruces en la imagen del polígono de $n$ segmentos ni siquiera se puede alcanzar cuando $n$ es impar. Aquí presentamos una mejoría a la cota anterior.

Primero, supongamos que renumeramos los vértices de $P$ en secuencia de manera que $v_{1}$ sea un punto en la frontera del núcleo convexo generado por los vértices de $P$. Por lo tanto, podemos encontrar un plano $\mathcal{P}_{1}$ que intersecte a $P$ únicamente en el vértice $v_{1}$, con $P$ ubicado completamente en un lado de $\mathcal{P}_{1}$.

Sea $\mathcal{S}$ una esfera grande centrada en $v_{1}$ que envuelva a todo $P$, y consideremos la imagen de la proyección radial $p: P-\left\{v_{1}\right\} \rightarrow \mathcal{S}$. A razón de nuestra selección de $v_{1}$, esta

\footnotetext{
11 Ver la Tabla 1 de [6].

12 Además de "minimal polygon index," otros autores en Inglés se refieren a este invariante como "stick number" $[1,8,10]$, "broken line number" [21] o simplemente "edge number" [19, 24].

13 Esta construcción aparece en la prueba del Teorema 7 de [21] y como el Ejercicio 1.38 de [1].
} 
imagen cae completamente en uno de los hemisferios de $\mathcal{S}$ definidos por el ecuador $\mathcal{S} \cap \mathcal{P}_{1}$. Además, notamos que $p$ lleva los interiores de los segmentos $v_{1} v_{2}$ y $v_{1} v_{n}$ respectivamente a los puntos $p\left(v_{2}\right)$ y $p\left(v_{n}\right)$. Así, al escoger un $P$ genérico en $\mathfrak{G e o}^{(n)}$, podemos asumir que $\Gamma=p\left(P-\left\{v_{1}\right\}\right)$ consiste de una cadena de $n-2 \operatorname{arcos}$ geodésicos en $\mathcal{S}$ los cuales se intersectan en cruces de valencia 4 .

Supongamos que $\Gamma$ tiene $c$ cruces. Cómo $\Gamma$ está contenido en un solo hemisferio de $\mathcal{S}$, cada par de arcos se intersecta solamente una vez. Además, arcos adyacentes no se pueden intersectar, así que cada uno de los $n-4$ arcos interiores $p\left(v_{3} v_{4}\right), \ldots, p\left(v_{n-2} v_{n-1}\right)$ puede intersectar a un máximo de $n-5$ arcos, mientras que cada uno de los arcos externos $p\left(v_{2} v_{3}\right)$ y $p\left(v_{n-1} v_{n}\right)$ intersecta a un máximo de $n-4$ arcos. Por lo tanto,

$$
c \leq \frac{1}{2}((n-4)(n-5)+2(n-4))=\frac{(n-3)(n-4)}{2} .
$$

Sea $\epsilon>0$ un número lo suficientemente pequeño para que la bola cerrada $\mathcal{B}_{\epsilon}$ de radio $\epsilon$ centrada sobre $v_{1}$ intersecte al polígono $P$ en exactamente dos pequeños subsegmentos de $v_{1} v_{2}$ y $v_{n} v_{1}$, como lo demuestra la Figura 19(a). Supongamos que el segmento $v_{1} v_{2}$ intersecta a la esfera $\partial \mathcal{B}_{\epsilon}$ en el punto $q_{1}$. Además, sea $q_{2}$ el punto donde el ecuador $\partial \mathcal{B}_{\epsilon} \cap \mathcal{P}_{1}$ intersecta al medio plano que contiene a $v_{2}$ y cuya frontera es la línea determinada por $v_{1}$ y $v_{3}$. Entonces podemos deformar el segmento $q_{1} v_{1}$ de manera que se encorve a lo largo de un arco geodésico $\alpha_{1}$ desde $q_{1}$ hasta $q_{2}$ y luego proceda en línea recta hasta $v_{1}$. Ver la Figura 19(b). Nótese que como el arco $\alpha_{1}$ se encuentra en el mismo plano que $v_{2} v_{3}$, $p\left(\alpha_{1}\right) \cup p\left(v_{2} v_{3}\right)$ forma una sola trayectoria geodésica sobre $\mathcal{S}$ desde $p\left(v_{3}\right)$ hasta $p\left(q_{2}\right)$. Por eso, nuestra cota superior sobre el número total de cruces es válida aún después de esta deformación.

De forma similar, sea $q_{3}$ el punto de intersección entre el ecuador $\partial \mathcal{B}_{\epsilon} \cap \mathcal{P}_{1}$ y el medio plano que contiene a $v_{n}$ y cuya frontera es la línea determinada por $v_{1}$ y $v_{n-1}$ y sea $q_{4}$ el punto en el cual el segmento $v_{n} v_{1}$ intersecta a $\partial \mathcal{B}_{\epsilon}$. Entonces el segmento $v_{1} q_{4}$ se puede deformar de manera que viaje en línea recta desde $v_{1}$ hasta $q_{3}$ y que de ahí siga un arco geodésico $\alpha_{3}$ hasta $q_{4}$. Ver la Figura 19(c). Como ocurrió anteriormente, el arco $\alpha_{3}$ está ubicado en el mismo plano que $v_{n-1} v_{n}$, por lo que $p\left(v_{n-1} v_{n}\right) \cup p\left(\alpha_{3}\right)$ forma una sola trayectoria geodésica sobre $\mathcal{S}$ desde $p\left(v_{n-1}\right)$ hasta $p\left(q_{3}\right)$. Por lo tanto, aún después de esta deformación, nuestra cota superior sobre el número de cruces es válida.

Finalmente, deformamos a $P$ al mover a $v_{1}$ hacia el interior del disco triangular $\triangle q_{2} v_{1} q_{3}$ mientras curvamos los segmentos $q_{2} v_{1}$ y $v_{1} q_{3}$ hasta que coincidan con un arco del ecuador $\partial \mathcal{B}_{\epsilon} \cap \mathcal{P}_{1}$, como en la Figura 19(d). Esta transformación final convierte a $P$ en una inmersión no-poligonal del mismo tipo (topológico) de nudo; la nueva inmersión coincide con $P$ fuera de la bola $\mathcal{B}_{\epsilon}$ pero completamente evita su interior. Mientras tanto, la imagen bajo $p$ de esta inmersión es simplemente una proyección (esférica) $\Gamma^{\prime}$ del nudo realizado por $P$, la cual consiste de los $n-2 \operatorname{arcos}$ de $\Gamma$ (con sus puntas extendidas por $p\left(\alpha_{1}\right)$ y $\left.p\left(\alpha_{3}\right)\right)$ junto con un $(n-1)^{\text {avo }}$ arco $\alpha_{2}$ que corre a lo largo del ecuador $\mathcal{S} \cap \mathcal{P}_{1}$ uniendo los puntos $p\left(q_{2}\right)$ y $p\left(q_{3}\right)$. Como $\Gamma$ se encuentra completamente a un lado del ecuador, $\alpha_{2}$ no cruza ningún otro arco. Por lo tanto, la nueva proyección no tiene más cruces de las que tenía antes de la última deformación, provando el siguiente teorema. 
Teorema 4. Supongamos que el nudo $K$ tiene un número mínimo de cruces $c(K)$ y un índice poligonal mínimo $p(K)$. Entonces

$$
c(K) \leq \frac{(p(K)-3)(p(K)-4)}{2} .
$$

Al completar el cuadrado en (6) obtenemos

$$
2 c \leq p^{2}-7 p+12=\left(p-\frac{7}{2}\right)^{2}-\frac{1}{4},
$$

o sea que

$$
p(K) \geq \frac{7+\sqrt{8 c(K)+1}}{2} .
$$

Notamos que el Teorema 4 predice correctamente que el trébol es el único nudo no trivial que se puede realizar con seis segmentos.

En el caso de los octágonos, la nueva cota sobre el número de cruces es 10. Sin embargo, en [4] sistemáticamente consideramos las posibles proyecciones $\Gamma^{\prime}$ de nudos que resultan de la deformación descrita anteriormente en la Figura 19 y de esta forma enumeramos los

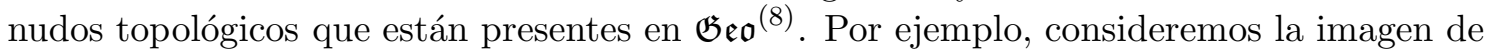
la proyección con diez cruces que aparece en la Figura 20(a). Al escoger apropiadamente en cada cruz cuál cuerda pasa por encima y cuál pasa por debajo, obtenemos una proyección $\Gamma^{\prime}$ del nudo que corresponde, como se indicó anteriormente, a un octágono $P$. Al hacer esta selección, tenemos ciertos puntos en mente:

(i) Si $v_{2} v_{3}$ pasa por debajo de todas sus cruces, entonces el interior del disco triangular $\triangle v_{1} v_{2} v_{3}$ no intersecta al resto de $P$. En este caso, se puede deformar $P$, empujando a $v_{2}$ en una trayectoria recta hacia el punto medio del segmento $v_{1} v_{3}$, hasta que $P$ coincida con un heptágono. Similarmente, si $v_{7} v_{8}$ pasa por debajo de todas sus cruces, existirá una isotopía que reduce a $P$ a un heptágono. Por lo tanto, no hay necesidad de considerar estos diagramas.

(ii) $\mathrm{Si}$ ambos de los segmentos $v_{5} v_{6}$ y $v_{6} v_{7}$ pasan por debajo de $v_{3} v_{4}$, como en la Figura 20(b), entonces podemos deformar a $P$ de manera que el $\Gamma^{\prime}$ correspondiente tenga dos cruces menos. Por ejemplo, podemos encoger las longitudes de $v_{5} v_{6} \mathrm{y}$ $v_{6} v_{7}$, en esencia reduciendo el diagrama con una movida Reidemeister de tipo II. En casos similares a éste, podemos ignorar las situaciones que se puedan reducir con este tipo de isotopía y posponer su análisis hasta que examinemos el diagrama reducido.

(iii) Ciertas selecciones de cruces nos llevan a configuraciones que son imposibles de realizar con segmentos rectos. Por ejemplo, consideremos las tres cruces seleccionadas en la Figura 20(c). Sea $\mathcal{P}$ el plano que contiene a $v_{4}, v_{5}$ y $v_{6}$. Notamos que el interior del segmento $v_{6} v_{7}$ se debe de encontrar completamente sobre el plano $\mathcal{P}$, ya que este segmento comienza en $v_{6}$ en el plano y luego cruza sobre $v_{4} v_{5}$. Similarmente, el interior del segmento $v_{3} v_{4}$ está ubicado completamente debajo del plano $\mathcal{P}$, pues este segmento cruza por debajo de $v_{5} v_{6}$ antes de terminar en $v_{4}$ en el plano. Esto significa que $v_{3} v_{4}$ no puede cruzar por encima de $v_{6} v_{7}$, como lo hace en la Figura 20(c), a menos de que uno de los dos segmentos esté torcido. 
(iv) La Figura 20(d) demuestra una selección inverosímil de cruces particularmente difícil. Este diagrama corresponde a una realización del nudo $8_{18}$, la cual se puede ver en la Figura 21. Aquí el problema no es tan obvio como antes. Es más, entre todas las configuraciones imposibles que encontramos en [4], ésta es la única que no es claramente imposible. Sin embargo, a través de un delicado argumento, incluyendo la introducción de auto-intersecciones y una cuidadosa cuenta de dimensiones, podemos demostrar que no hay manera de construir esta configuración. Los detalles de este argumento aparecerán en un artículo próximo.

Después de considerar todas las posibles proyecciones $\Gamma^{\prime}$ con más de seis cruces, encontramos que los nudos $8_{19}$ y $8_{20}$ son los únicos con índice poligonal de ocho y número de cruces mayor que seis. Como ya se sabe que existen realizaciones octogonales de todo nudo $K$ con número mínimo de cruces $c(K) \leq 6$, obtenemos la lista completa de los nudos topológicos presentes en $\mathfrak{G}_{\mathfrak{e o}}{ }^{(8)}$, como lo indica el Teorema 1(iv). Con la excepción de $6_{3}$, el nudo cuadrado $3_{1}-3_{1}$, el nudo doble $4_{1}$, y el nudo trivial, todo nudo en esta lista es quiral y por lo tanto contribuye a por lo menos dos arco-componentes de $\mathfrak{G}_{\mathfrak{e o}}{ }^{(8)}$. Por esta

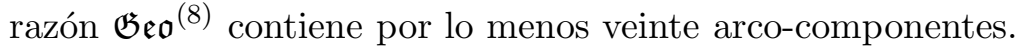

\section{Reconocimientos}

Quisiera agradecer a Ken Millett, quien alegremente ha compartido conmigo su consejo, su perspicacia y sus preguntas más difíciles desde mis primeros años de estudio de posgrado. Además, le agradesco a la facultad de la Escuela de Matemáticas de la Universidad de Costa Rica, especialmente a Asdrúbal Duarte y a Bernardo Montero, por su hospitalidad durante mi estadía en San José en 1999. Espero que ésta sea solamente la primera de muchas aventuras juntos. Finalmente, quiero dedicarle esta obra a toda mi familia, en Costa Rica y en los Estados Unidos; gracias a ellos he tenido la oportunidad de no sólo seguir mis sueños sino de poder hacerlo en dos mundos diferentes.

\section{Referencias}

1. Adams, C. C. (1994) The Knot Book: An Elementary Introduction to the Mathematical Theory of Knots. W. H. Freeman and Co., New York.

2. Alexander, J. W.; G. B. Briggs (1927) "On types of knotted curves", Annals of Mathematics 28: $562-586$.

3. Babini, J. (1952) Historia Sucinta de la Matemática, Colección Austral, Espasa-Calpe Argentina, S.A., Buenos Aires, Argentina.

4. Calvo, J. A. (1998) Geometric Knot Theory: The Classification of Spatial Polygons with a Small Number of Edges. Ph.D. Thesis, University of California, Santa Barbara.

5. Calvo, J. A. (2000) "The embedding space of hexagonal knots", Preprint, North Dakota State University, Fargo, ND. (Aparecerá en Topology and its Applications 110(2).)

6. Calvo, J. A.; Millett, K. C. (1999) "Minimal edge piecewise linear knots", Ideal Knots, A. Stasiak, V. Katrich y L. H. Kauffman (Eds.), Series on Knots and Everything, vol. 19, World Scientific, Singapore: $107-128$.

7. Curcio Rufo, Q. La Historia de Alejandro Magno (Historiarium Alexandri Magni Macedonis Libris), libro III, capítulo II.

8. Adams, C. C.; Brennan, B. M.; Greilsheimer, D. L.; Woo, A. K. (1997) "Stick numbers and composition of knots and links", Journal of Knot Theory and its Ramifications 6(2): 149-161.

9. Gordon, C. McA.; Luecke, J. (1989) "Knots are determined by their complements", Bulletin of the American Mathematical Society (New Series) 20 (1): 83-87. 
10. Furstenberg, E.; Lie, J.; Schneider, J. (1998) "Stick knots", Chaos, Solitons and Fractals 9(4-5): 561-568.

11. Jones, V. F. R. (1985) "A polynomial invariant for knots via von Neumann algebras", Bulletin of the American Mathematical Society (New Series) 12(1): 103-111.

12. Freyd, P.; Yetter, D.; Hoste, J.; Lickorish, W. B. R.; Millett, K. C.; Ocneanu, A. (1985) "A new polynomial invariant of knots and links", Bulletin of the American Mathematical Society (New Series) 12(2): 239-246.

13. Hoste, J.; Thistlethwaite, M.; Weeks, J. (1998) The first 1,701,936 knots, Mathematical Intelligencer 20(4): $33-48$.

14. Kauffman, L. H. (1987) On Knots, Annals of Mathematics Studies, vol. 115, Princeton University Press, Princeton, NJ.

15. Kirkman, T.P. (1885) "The enumeration, description and construction of knots with fewer than 10 crossings", Transactions of the Royal Society of Edinburgh 32: 281-309.

16. Lickorish, W. B. R. (1962) "A representation of orientable combinatorial 3-manifolds", Annals of Mathematics (Second Series) 76: 531-540.

17. Lickorish, W. B. R., An Introduction to Knot Theory, Graduate Texts in Mathematics, vol. 175, Springer, New York.

18. Litle, C. N. (1885) "On knots, with a census of order ten", Transactions of the Connecticut Academy of Science 18: 374-378.

19. Meissen, M. (1998) "Edge number results for piecewise-linear knots", Knot Theory, Polish Academy of Sciences, Warsaw: 235-242.

20. Millett, K. C. (1994) "Knotting of regular polygons in 3-space", Journal of Knot Theory and its Ramifications 3(3): 263-278; también en Random Knotting and Linking, K. C. Millett y D. W. Sumners (Eds.), Series on Knots and Everything, vol. 7, World Scientific, Singapore: 31-46.

21. Negami, S. (1991) "Ramsey theorems for knots, links, and spatial graphs", Transactions of the American Mathematical Society 324(2): 527-541.

22. Randell, R. (1988) "A molecular conformation space", MATH/CHEM/COMP 1987, R. C. Lacher (Ed.), Studies in Physical and Theoretical Chemistry, vol. 54, Elsevier Science, Amsterdam: 125-140.

23. Randell, R. (1988) "Conformation spaces of molecular rings", MATH/CHEM/COMP 1987 R. C. Lacher (Ed.), Studies in Physical and Theoretical Chemistry, vol. 54, Elsevier Science, Amsterdam: 141-156.

24. Randell, R. (1994) "An elementary invariant of knots", Journal of Knot Theory and its Ramifications 3(3): 279-286; también en Random Knotting and Linking, K. C. Millett y D. W. Sumners (Eds.), Series on Knots and Everything, vol. 7, World Scientific, Singapore: 47-54.

25. Reidemeister, K. (1983) Knot Theory, traducido del alemán y editado por L. F. Boron, C. O. Christenson y B. A. Smith, BCS Associates, Moscow, ID.

26. Rolfsen, D. (1976) Knots and Links, Mathematical Lecture Series, vol. 7, Publish or Perish, Houston, TX.

27. Tait, P. G. (1898) "On knots, I, II, III", Scientific Papers, vol. 1, Cambridge University Press, Cambridge: $273-347$.

28. Thurston, W. P. (1982) "Three-dimensional manifolds, Kleinian groups and hyperbolic geometry", Bulletin of the American Mathematical Society (New Series) 6(3): 357-381.

29. Thurston, W. P. (1986) "Hyperbolic structures on 3-manifolds. I. Deformation of acylindrical manifolds", Annals of Mathematics (Second Series) 124(2): 203-246.

30. Thurston, W. P. (1997) Three-Dimensional Geometry and Topology. Vol. 1, editado por S. Levy, Princeton University Press, Princeton, NJ.

31. Tutton, A. E. H. (1922) Crystallography and Practical Crystal Measurement, vol. 1, Macmillan and Co. Ltd., London.

32. Wallace, A. H. (1960) "Modifications and cobounding manifolds", Canadian Journal of Mathematics 12: 503-528.

33. Whitney, H. (1967) "Elementary structure of real algebraic varieties", Annals of Mathematics 66: 545-556.

34. Ziegler, G. M. (1995) Lectures on Polytopes, Graduate Texts in Mathematics, vol. 152, Springer Verlag, New York. 

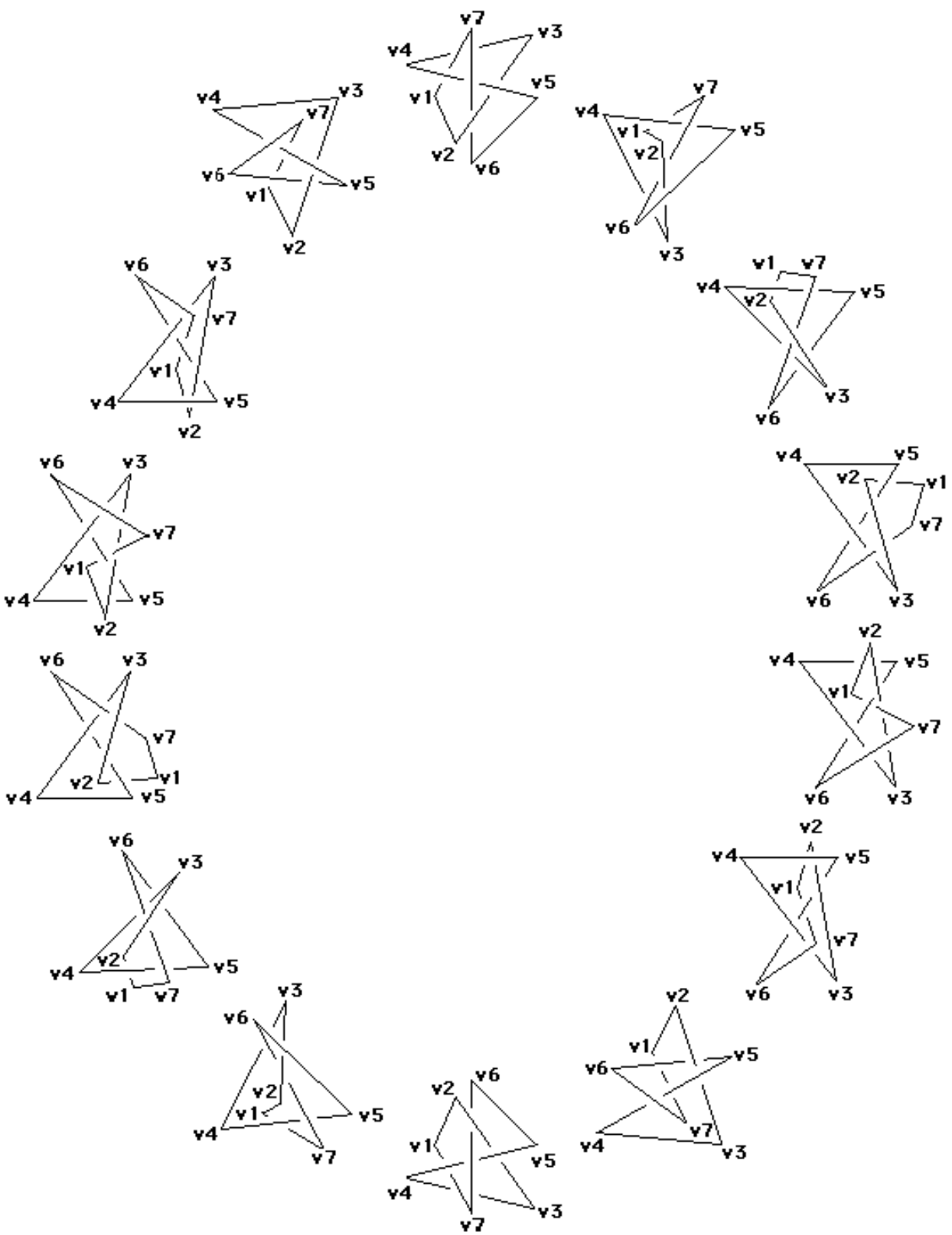

FigurA 18. Los nudos dobles heptagonales son aquirales. 


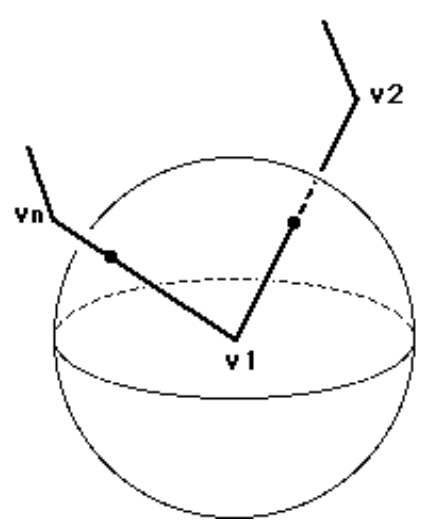

(a)

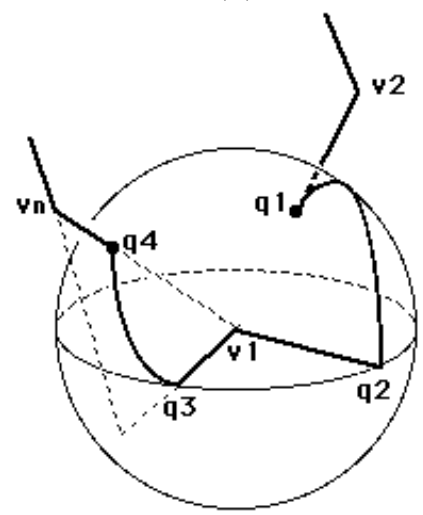

(c)

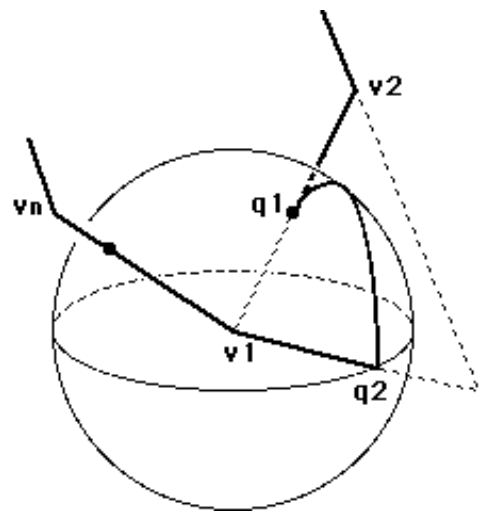

(b)

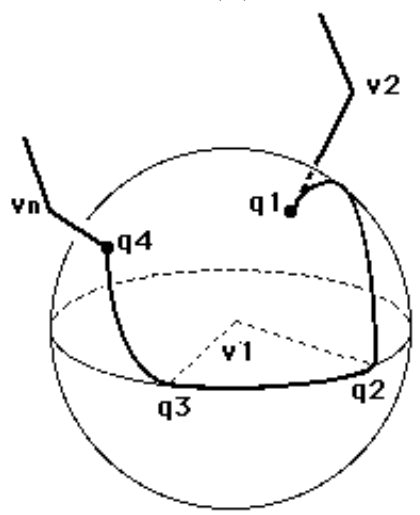

(d)

Figura 19. La deformación de $P$ dentro de una pequeña bola de radio $\epsilon$ alrededor de $v_{1}$. 


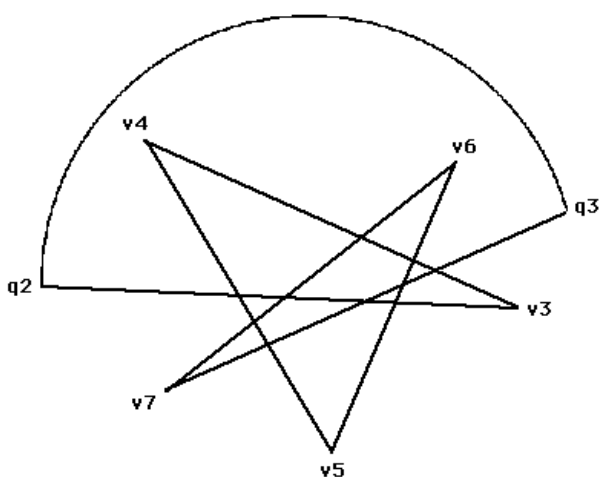

(a)

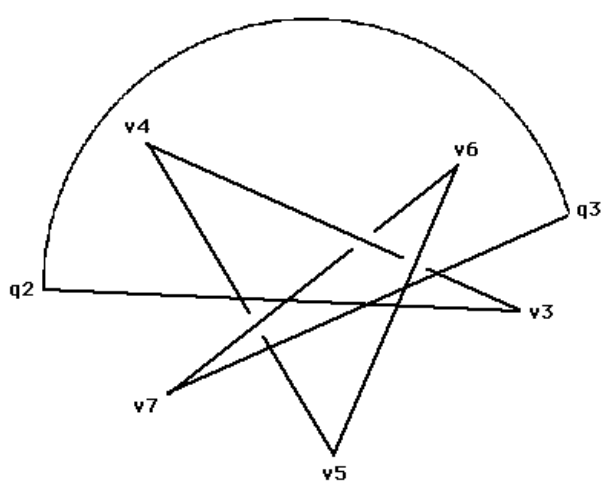

(c)

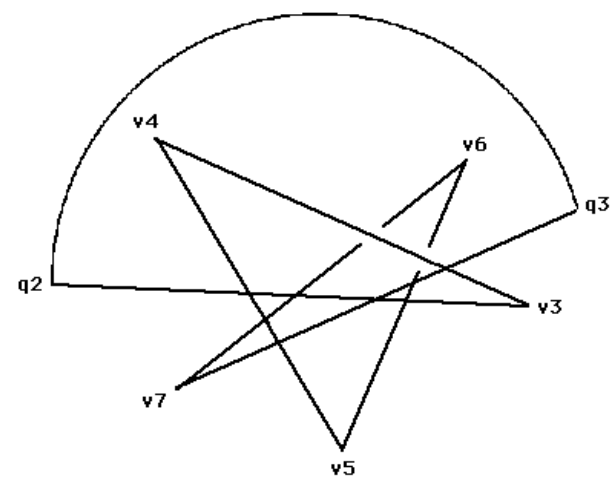

(b)

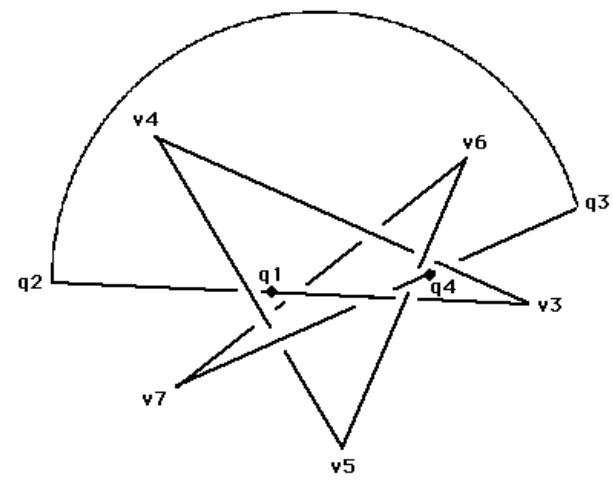

(d)

FiguRA 20. La imagen de una proyección con diez cruces.

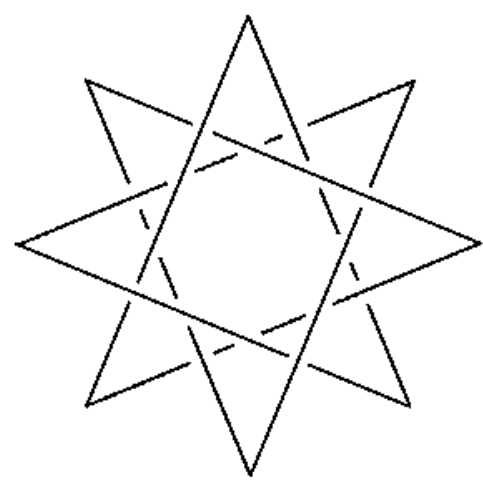

Figura 21. Esta inmersión octogonal del nudo $8_{18}$ no se puede construir a base de segmentos rectos. 